\title{
South China Sea Internal Tide/Internal Waves- Impact on the Temporal Variability of Horizontal Array Gain at $276 \mathrm{~Hz}$
}

\author{
Marshall H. Orr, Bruce H. Pasewark, Associate Member, IEEE, Stephen N. Wolf, \\ James F. Lynch, Senior Member, IEEE, Theodore Schroeder, and Ching-Sang Chiu
}

\begin{abstract}
The temporal variability of the spatial coherence of an acoustic signal received on a bottomed horizontal array has been calculated for $276-\mathrm{Hz}$ narrow-band signals. A conventional plane wave beamformer was applied to the received signals. The temporal variability of the array's omnipower, beam power, and array gain are related to variability in the sound-speed field. The spectral characteristics of array omnipower are nonstationary and changed as the spectral characteristics of the temperature field varied. The array omnipower and beam-power variability tracked each other in time and varied by as much as $15 \mathrm{~dB}$ over time intervals as short as $7 \mathrm{~min}$. Array gain varied up to $5 \mathrm{~dB}$ and usually tracked the omnipower variability. A contiguous 24-h section of data is discussed in detail. This data section is from a time period during which the high-frequency fluid dynamic perturbation of the sound-speed field was of smaller amplitude than other sections of the 16-d data set. Consequently, this section of data sets an upper bound for the realizable array gain. The temporal variability of array gain and spatial coherence at times appears to be correlated with environmental perturbation of the sound-speed field, but are also correlated with changes in the signal-to-noise ratio. The data was acquired during the Office of Naval Research's South China Sea Asian Seas International Acoustics Experiment. The 465-m 32-channel horizontal array was placed on the bottom in $120 \mathrm{~m}$ of water at the South China Sea shelf break. The acoustic source was moored in $114 \mathrm{~m}$ of water $\sim 19 \mathrm{~km}$ from the receiving array.
\end{abstract}

Index Terms-Array signal gain, coherence, internal waves, narrow-band conventional beamforming, shallow-water arrays.

\section{INTRODUCTION}

$\mathbf{T}$ HE spatial and temporal properties of acoustic signals recorded on a bottomed horizontal array during the South China Sea Asian Seas International Acoustic Experiment (ASIAEX) will be overviewed. The primary focus of this paper will be on the response of a narrow-band $(276 \mathrm{~Hz})$ conventional beamformer to environmental perturbation of the sound-speed field and variability in the signal-to-noise ratio (SNR).

The South China Sea component (April-May 2001) of the Office of Naval Research's (ONR) ASIAEX was an interdisci-

Manuscript received October 1, 2003; revised November 24, 2003. This work was supported by the Office of Naval Research. This paper is a WHOI contribution.

M. H. Orr, B. H. Pasewark, and S. N. Wolf are with The Naval Research Laboratory, Washington, DC 20375 USA (e-mail: orr@wave.nrl.navy.mil).

J. F. Lynch is with The Woods Hole Oceanographic Institution, Woods Hole, MA 02543 USA.

T. Schroeder is on active duty with the U.S. Navy.

C.-S. Chiu is with The Naval Postgraduate School, Monterey, CA 93943-5001 USA.

Digital Object Identifier 10.1109/JOE.2004.836794

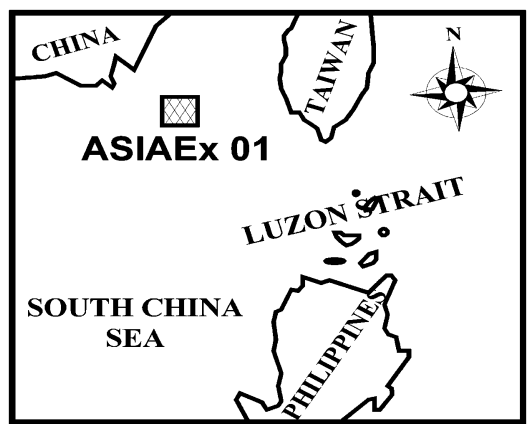

Fig. 1. ASIAEX 01 South China Sea component operational site, located on the continental shelf $177 \mathrm{~nm}$ east of Taiwan and $80 \mathrm{~nm}$ southeast of mainland China.

plinary physics of acoustic propagation, physical oceanography, and marine geology and geophysics experiment. The acoustic experiment had two components. The first was the study of the physics of acoustic signal propagation in a dynamic shelf/slope sound-speed field. The second focused on the characterization of the temporal variability of the local ambient noise field. Research scientists from Singapore, Taiwan, and the United States participated in the study.

The experiment site (Fig. 1) was deliberately chosen because it was known to be one of the world's more energetic shelf-break environments due to the passage environments. Internal tides and internal waves generated both at the Luzon Straits and near the experiment site were expected to cause significant perturbation of the sound-speed field. The internal waves generated in the Luzon Straits were expected to be nonlinear and were hypothesized to undergo depression to elevation conversion and dissipation in the vicinity of the shelf break [1].

\section{EXPERIMENT}

In brief, acoustic signals propagating between fixed sources and receivers were detected and recorded for $16 \mathrm{~d}$. A vertical and horizontal array were moored (Fig. 2) near the South China Sea shelf break. Acoustic sources were moored down- and crossslope from the arrays. Sound-speed field variability in the vicinity of the experiment was quantified over spatial and temporal scales ranging from tens of kilometers to tens of meters and from days to seconds using a variety of oceanographic instrumentation, including: SeaSoar, thermistor string moorings, a tow-yo conductivity, temperature, and depth (pressure) (CTD) sensor package, and temperature and pressure sensors attached to the acoustic sources and acoustic receiving arrays [2]. To support the interpre- 


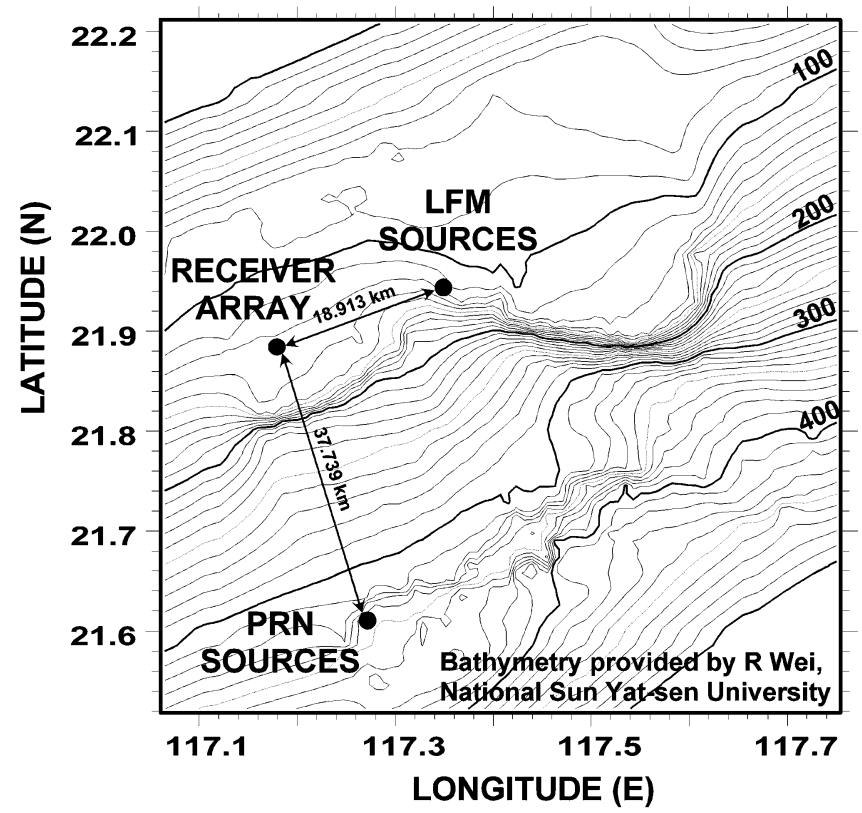

Fig. 2. Moored LFM sources, $224 \mathrm{~Hz}$ PRN source, and acoustic receiver array locations, together with the ASIAEX 01 South China Sea site bathymetry.

tation of the acoustic data, bottom variability was measured and subbottom geoacoustic properties were estimated from cores and via geoacoustic inversions [3], [4].

\section{A. Acoustic Sources and Receivers}

Acoustic signals were detected using a 16-channel vertical line array (VLA) and a 32-channel horizontal line array (HLA) from the Naval Postgraduate School (NPGS), Monterey, CA, and recorded using an autonomous 48-channel recording system from the Woods Hole Oceanographic Institution (WHOI), Woods Hole, MA. The VLA was moored in $\sim 125 \mathrm{~m}$ of water (Fig. 2) and had 16 hydrophones distributed between the 42- and 121-m depth interval. The top ten hydrophones were spaced at $3.75 \mathrm{~m}$ and the bottom six hydrophones were spaced at $7.5 \mathrm{~m}$. The $465-\mathrm{m}$ HLA had 32 equally spaced hydrophones (i.e., 15-m hydrophone separation). One end of the HLA was located $2 \mathrm{~m}$ from the VLA mooring and the other end was located approximately $400 \mathrm{~m}$ away at a bearing of $224^{\circ} \mathrm{T}$. The HLA was placed so that no moored acoustic sources were in the end fire beams of the array.

Two linear frequency-modulated Naval Research Laboratory sources were placed cross-slope from the receiving arrays. One operated at a $300-\mathrm{Hz}$ center frequency, the other at a $500-\mathrm{Hz}$ center frequency. A pseudorandom noise (PRN) sequence was generated by a WHOI 224-Hz source located downslope (Fig. 2) and two NPGS 400-Hz sources, one located downslope and one located cross-slope. The WHOI 224-Hz source and the NRL $300-$ and $500-\mathrm{Hz}$ sources were used to navigate the horizontal line array. The $300-\mathrm{Hz}$ NRL source was moored at a depth of $102 \mathrm{~m}$ in $114 \mathrm{~m}$ of water $\sim 18.9 \mathrm{~km}$ from the VLA receiver. The NRL 500-Hz LFM source was moored at a depth of 100 $\mathrm{m}$ in $112 \mathrm{~m}$ of water, $\sim 18.4 \mathrm{~km}$ from the VLA receiver. The LFM signals were projected with a 50\% duty cycle (i.e., $2.048 \mathrm{~s}$ on with a $10 \%$ taper, $2.048 \mathrm{~s}$ off). The $300-\mathrm{Hz}$ LFM signal had a $60-\mathrm{Hz}$ bandwidth and the $500-\mathrm{Hz}$ LFM signal had a $90-\mathrm{Hz}$

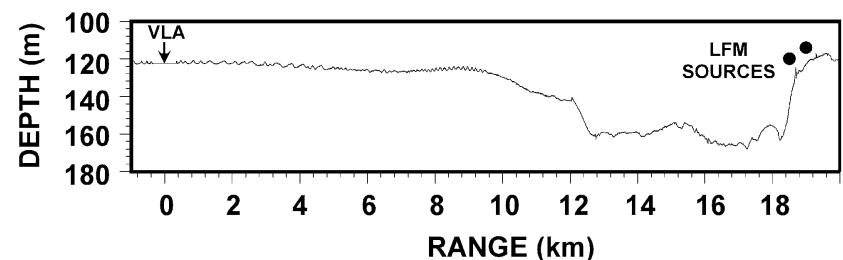

Fig. 3. Bathymetry of the crossslope acoustic propagation path. The acoustic VLA is located at range 0 and the LFM sources are located at ranges 18.4 $(500 \mathrm{~Hz})$ and $18.9 \mathrm{~km}(300 \mathrm{~Hz})$. The bathymetry was provided by S. G. Schock, The Florida Atlantic University, Boca Raton, FL.

bandwidth. Both sources used rubidium clocks and had nominal source levels of 181-dB re $1 \mu \mathrm{Pa} @ 1 \mathrm{~m}$. The 224-Hz WHOI source was moored at a depth of $331 \mathrm{~m}$ in $345 \mathrm{~m}$ of water and was located $\sim 37.7 \mathrm{~km}$ downslope from the VLA receiver. It projected a 3.9375-s 63-digit 224-Hz PRN sequence with a 16-Hz bandwidth. Thirty PRN sequences were transmitted in sequence (total time $=118.125 \mathrm{~s}$ ) every $300 \mathrm{~s}$, resulting in a $40 \%$ duty cycle. The 224-Hz PRN projector had a source level of $182 \mathrm{~dB}$ re $1 \mu \mathrm{Pa} @ 1 \mathrm{~m}$. All sources were moored in high tension to minimize source motion in variable currents. The two NPGS $400-\mathrm{Hz}$ PRN signal properties will not be addressed.

\section{B. Acoustic Environment}

The acoustic experiment was conducted in a stratified water column that was perturbed by large currents caused by the tide, internal tides, and associated internal wave fields. Ship-radiated noise and bottom currents caused SNR variability at the receiving arrays.

The currents physically moved both the vertical and horizontal arrays. The vertical array was tilted on occasion by more than $14^{\circ}$. Acoustic travel-time measurements indicate that the HLA was not straight and that the HLA hydrophones were moved tens of meters every 6-12 h. The horizontal array elements were navigated using a technique developed by S. Wolf of the NRL. Appendix I outlines the array navigation procedure and discusses the time history of the array element locations. The horizontal array motion induced large noise events as the hydrophones were dragged over the bottom. In addition, there appeared to be noise events on the horizontal array elements related to bottom current flow over the array or movement of gravel or sand past the hydrophones.

The noise radiated from a fishing vessel that served as a guard boat to protect the acoustic receiving assets from being damaged by the local fishing fleet is present throughout the data set. The guard boat spent a significant time maneuvering in the immediate vicinity of the acoustic arrays. As a result, the SNRs were lowered and our ability to unambiguously interpret the data in the context of the impact of the sound-speed variability was compromised.

\section{Bottom and Subbottom Properties}

The bottom composition along the propagation path was variable, ranging from coarse sand and gravel to fine silts and clays. Several cores were taken by Taiwanese scientists and core analysis was done at NRL [3]. In addition, bottom compressional wave speed and attenuation were estimated from nadir incident (i.e., transducer orientedwith beam incident perpendicular to the ocean bottom) chirp sonar data by Shock [4]. Significant sand 


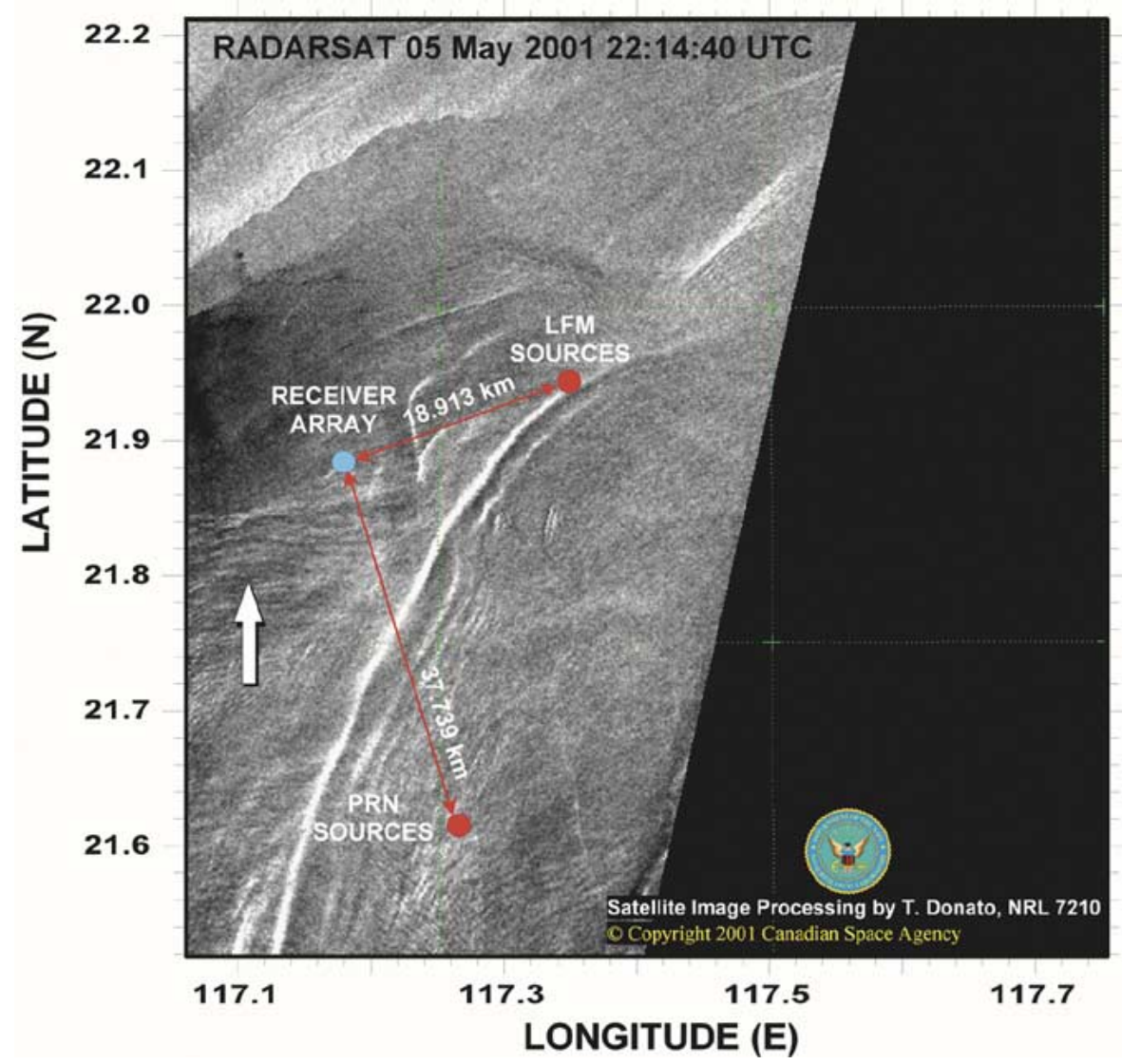

Fig. 4. SAR satellite image of an internal wave soliton arriving at the South China Sea shelf break. The prominent wave packet, white reflection curving from the sources to the southeast, was generated in the Luzon Straits. The SAR image was processed by T. Donato, Code 7210, NRL.

wave structure roughened the bottom. This is evident along the shelf propagation path, i.e., the $0-8-\mathrm{km}$ section of Fig. 3. The wave-number vector and spectra of the sand waves is unknown. Optical attenuation measurements by Gawarkiewicz [5] indicates active suspension of sediments from the bottom during internal tide and internal wave passage. This in turn suggests the sand waves are active and that the variability in the bottom roughness will be time dependent. Time dependence in bottom roughness will eventually have to be included in acoustic propagation modeling for the area.

\section{Oceanographic Environment}

1) Sound-Speed Field Perturbation by the Tide, Internal Tide, and Internal Wave Packets: As mentioned, the volumetric sound-speed field at the experiment site was continuously perturbed by currents associated with the tide as well as displacement of the pycnocline by the internal tide and internal wave packets generated by flow-over bathymetry at both the Luzon Straits and in the immediate vicinity of the experiment.

Nonlinear internal gravity wave packets generated in the Luzon Straits periodically propagated through the acoustic propagation paths. The packet-arrival interval changed from roughly semidiurnal to diurnal during the spring to neap tidal cycle [Fig. 5(g) and (h)]. The packets took about $4.5 \mathrm{~d}$ to propagate across the Luzon Basin to the South China Sea shelf break [2]. At the acoustic experiment site, synthetic aperture radar (SAR) imagery (Fig. 4) detected the Luzon Basin internal wave packets propagating upslope. Satellite-borne synthetic aperture radar (Fig. 4) and ship radar detected the surface expression of the wave packets as they propagated upslope. The packets had significant curvature. As a result, they reached the $300-$ and $500-\mathrm{Hz}$ acoustic sources approximately $150 \mathrm{~min}$ before arriving at the acoustic receiving arrays. The wave front curvature means that the acoustic signals propagated obliquely through the packet. Taking into account the curvature of the leading edge of the internal wave packet, the average upslope speed of propagation between the acoustic source and receiver is estimated to be $1.4 \mathrm{~m} / \mathrm{s}$. The propagation speed depends upon the water depth and stratification. Consequently, the instantaneous speed of the wave packet is expected to be variable as it propagates upslope. For instance, the speed of the surface expression of the Luzon Straits related internal wave packets was on the order of $2 \mathrm{~m} / \mathrm{s}$ in 260-300-m water depths.

The SAR image shows two surface expressions near the 300and $500-\mathrm{Hz}$ acoustic sources and several surface expressions near the $37-\mathrm{km}$ upslope acoustic propagation path. The image suggests the internal wave perturbation of the sound-speed field near the $300-$ and $500-\mathrm{Hz}$ acoustic sources will be simpler than along the upslope path. However, acoustic flow visualization records show that the internal wave packets near the sources extend at least $11 \mathrm{~km}$ downslope with multiple depth perturbations along the propagation direction [6] of the internal waves when the SAR showed only two surface expression separated by $1-2 \mathrm{~km}$. Consequently, SAR images may identify the presence of internal waves, but may not be useful from the standpoint of 


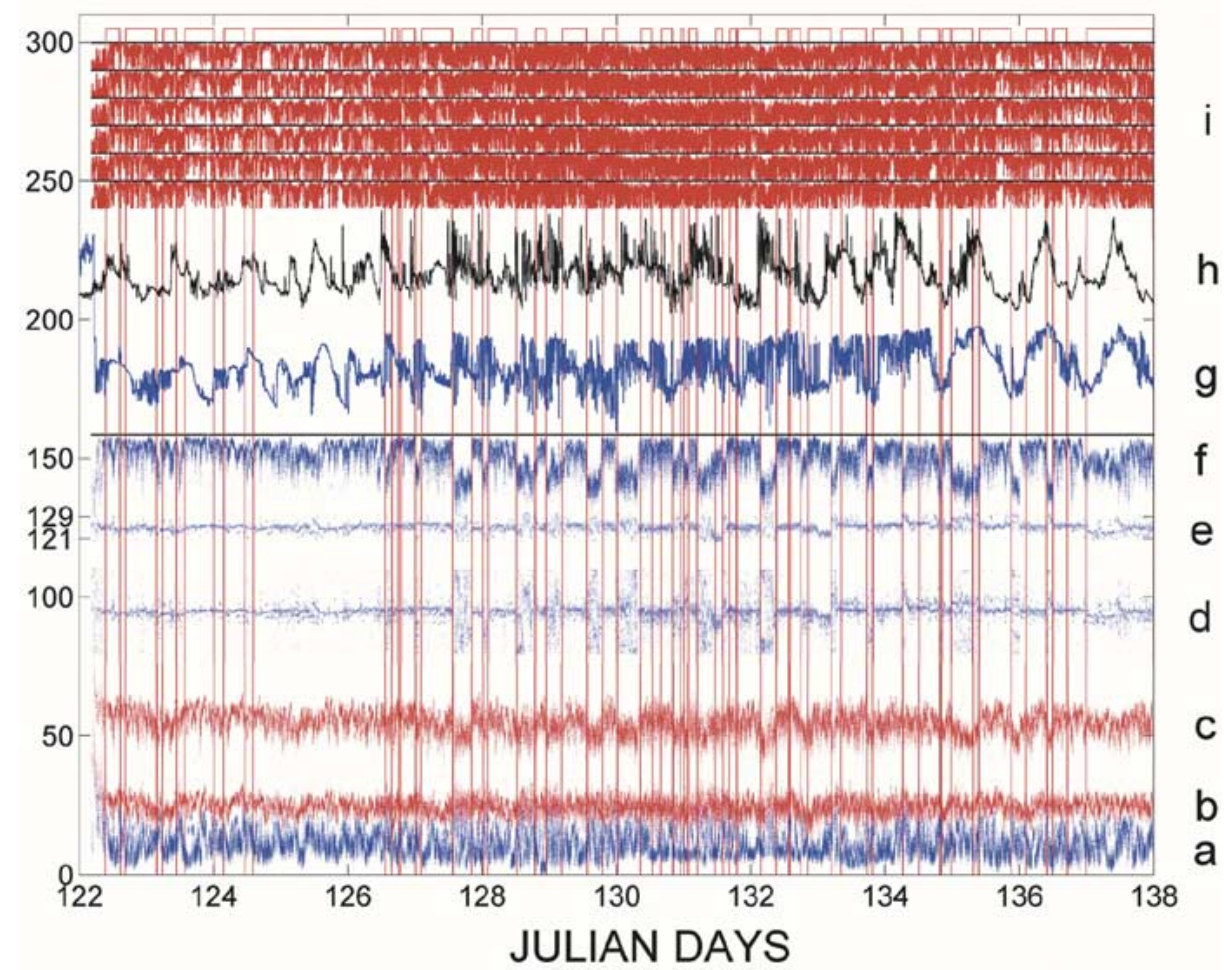

Fig. 5. Sixteen-day (JD 122-138) narrow-band 276-Hz array response and temperature record composite. (a) Omninoise, (b) omnipower, (c) beam power, (d) raw direction to $276 \mathrm{~Hz}$ acoustic source, (e) direction to acoustic source with noise removed, (f) array gain, (g) 37-m depth temperature record at the vertical line array, (h) 57-m depth temperature record near the sources, and (i) temporal variability of the spatial coherence for horizontal array phones 1 and 5,1 and 10,1 and 15,1 and 20, 1 and 25, and 1 and 29, respectively. The $y$-axis has decibel units when referring to omnipower, omninoise, and beam power. In the case of array gain, four units of $\mathrm{dB}=1 \mathrm{~dB}$ of array gain. Absolute temperature scale is presented in Fig. 12(a) and (b). In the direction to acoustic source with noise removed [Fig. 5(e)], 121 to 129 represents $\pm 4^{\circ}$. In the coherence plots (i), ten units of the $y$-axis represents a change in coherence from 0 to 1 .

accurately estimating the impact of the internal waves on the sound-speed field variability.

One of the locally generated wave packets (Fig. 4, arrow) is seen to the south of the receiving array. It has a wave front oriented about $75^{\circ}$ to the downslope acoustic propagation path. Its presence indicates that, at any time, the sound-speed field along the acoustic propagation path can be perturbed by multiple internal wave packets, each propagating in different directions. As a result, relating acoustic signal properties to specific fluid processes, e.g., Luzon Straits generated internal wave packet, must be approached with care because the separation $(\sim 18 \mathrm{~km})$ of the vertical temperature strings located at the vertical acoustic line array and the sound sources is too large to detect the presence of the internal wave field with the $75^{\circ}$ propagation vector when it is between the acoustic source and receiver arrays.

2) Temperature Variability: Continuous temperature and pressure records were taken simultaneously with the acoustic data by Duda and Irish [7]. A thermistor string was moored near the $300-$ and $500-\mathrm{Hz}$ sources and several temperature pods were mounted on the VLA. Temperature time series from each of the locations are presented [Figs. 5, 6, 10(g) and (h), and 12]. Since temperature dominated the sound-speed field, the temperature records will be used to infer sound-speed field variability.

Visual inspection of the 16-d temperature time series [Fig. 5(g)] reveals that the spectral composition is not constant in time. The data can be divided into three time sections, each having different spectral and energy content. The first and third sections, from Julian Day (JD) 122 to JD 127 and from JD 133 to JD 138, are dominated by the diurnal tide. Small amplitude short-period internal waves were present in both of these time intervals. The second section, from JD 127 to JD 133, has large-amplitude high-frequency temperature variability with a semidiurnal period.

The first $8 \mathrm{~d}$ of the composite acoustic and temperature records are shown at a larger scale in Fig. 6(g) and (h). This record encompasses the small-amplitude low-frequency temperature variability and a time period of equal length, during which the temperature fluctuations have a high-frequency larger amplitude internal wave component.

The power spectra of the temperature profiles at the source [Fig. 7(c1)] and acoustic receiver [Fig. 7(a1)] array for the 8-d measurement period are different from each other. The source location is more energetic and has a distinctly different slope at the shorter periods. Note that the long period spectral components were deliberately plotted clipped to emphasize the short-period variability that is of greater interest from the standpoint of the short-period acoustic signal and array-gain fluctuations.

The spectra for the two 4-d subsections [Fig. 7(a2) and (a3) and Fig 7(c2) and (c3)] have a different slope at both locations and in each time period. These spectra indicate that the statistical behavior of the temperature field is not stationary. In the next section, these spectra will be compared to the spectrum for the incoherent sum of the acoustic signals [Fig. 7(b)] received on 29 elements of the horizontal array. 


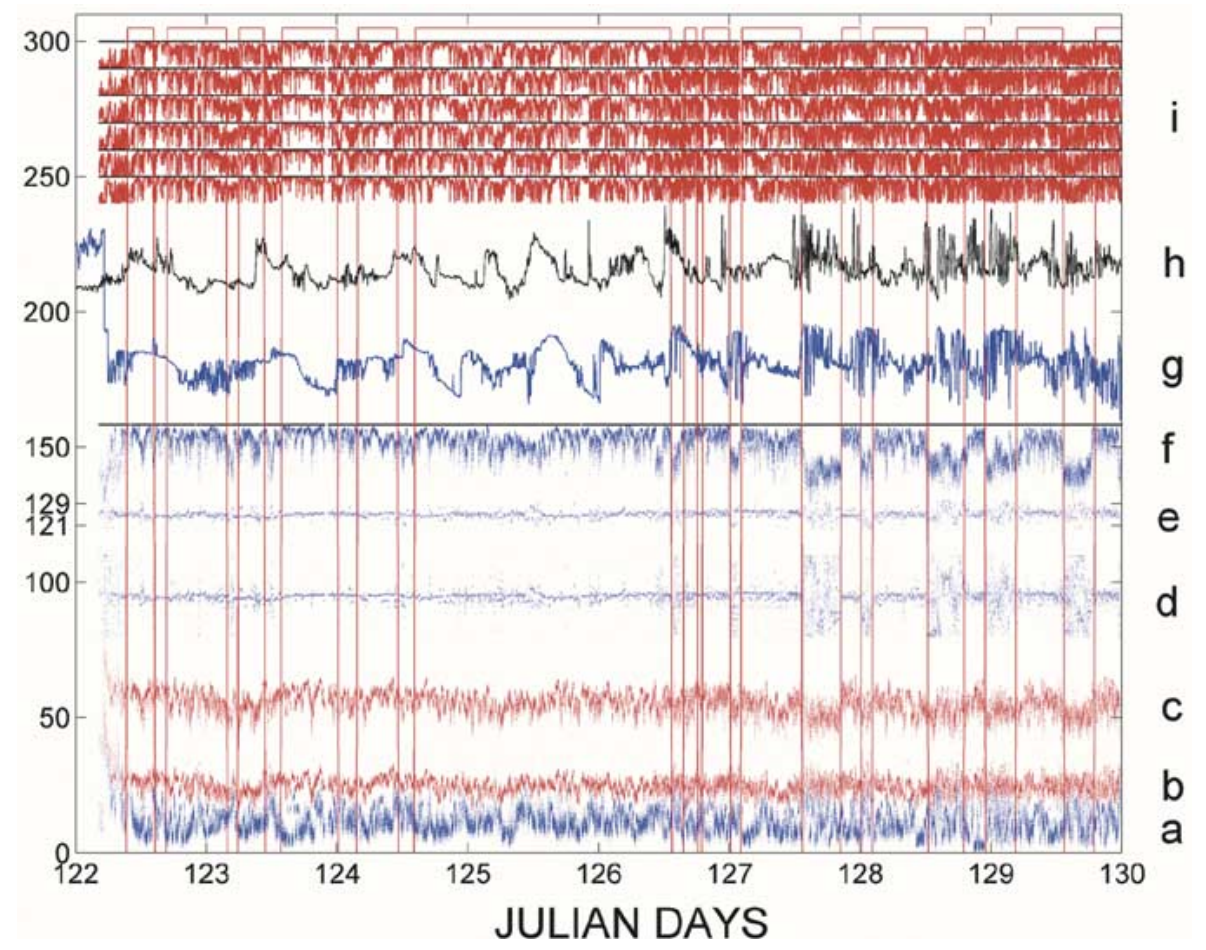

Fig. 6. Eight-day (JD 122-130) narrow-band 276-Hz array response and temperature record composite. (a) Omninoise, (b) omnipower, (c) beam power, (d) raw direction to $276-\mathrm{Hz}$ acoustic source, (e) direction to acoustic source with noise removed, (f) array gain, (g) 37-m depth temperature record at the vertical line array, (h) 57-m depth temperature record near the sources, and (i) temporal variability of the spatial coherence for horizontal array phones 1 and 5, 1 and 10, 1 and 15, 1 and 20, 1 and 25, and 1 and 29, respectively. The $y$-axis has decibel units when referring to omnipower, omninoise, and beam power. In the case of array gain, four units of $\mathrm{dB}=1 \mathrm{~dB}$ of array gain. The absolute temperature scale is presented in Fig. 12(a) and (b). The direction to the acoustic source [Fig. 6(e)] with noise removed, 121-129 represents $\pm 4^{\circ}$. In the coherence plots (i), ten units of the $y$-axis represents a change in coherence from 0 to 1 .
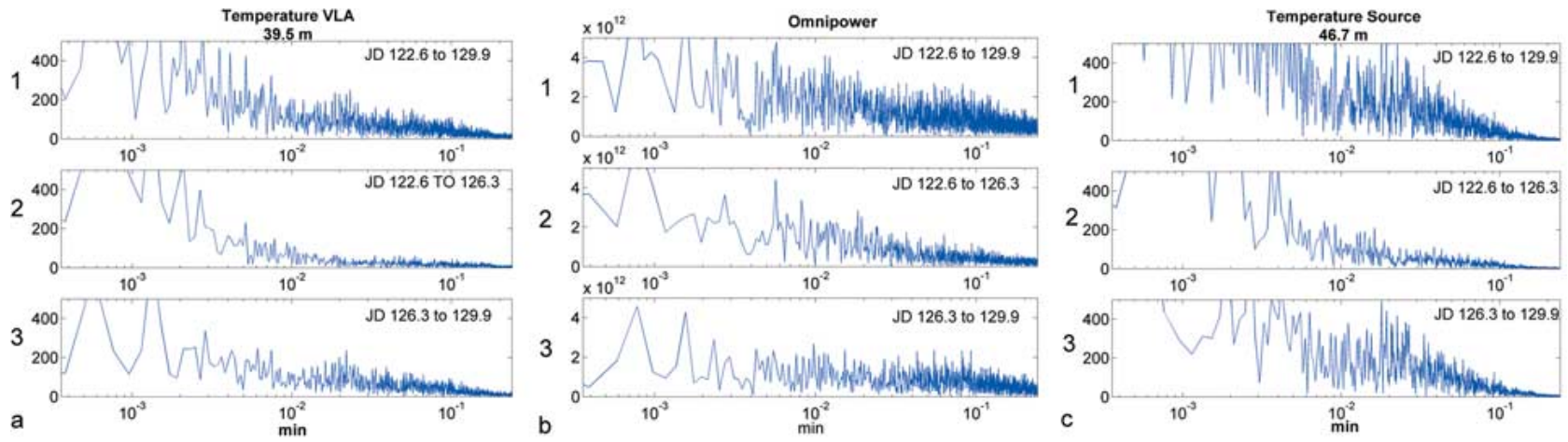

Fig. 7. Power spectra of omnipower and temperature for an 8-d time series and two 4-d subsets. The first 4-d subset corresponds to a time interval of lesser internal wave activity. Spectra of temperature and omnipower are nonstationary.

\section{Horizontal ARray Performance AND ACOUSTIC Signal PROPERTIES}

One of the objectives of the ASIAEX South China Sea experiment was to relate variability in acoustic signal properties and horizontal array performance to fluid-induced sound-speed variability. Initial analysis of the 16-d data set has resulted in a preliminary quantification of the following:

- temporal variability of the intensity fluctuations of acoustic signals received on a bottomed hydrophone;

- temporal variability in the temporal correlation of signals received on bottomed hydrophones;
- temporal variability of the horizontal array signal spatial coherence;

- temporal variability in array omnipower, beam power, array gain, and beam-angle wander.

Selected sections of the analysis will be presented. The focus will be on the response of a conventional narrow-band beamformer applied to $276-\mathrm{Hz}$ data acquired with the bottomed horizontal array.

Three composite figures showing 16-, 8-, and 1-d conventional beamformer response and its temporal variability are presented in Figs. 5, 6, and 10, respectively. Response of a convention beamformer with omnipower to omninoise power SNR 
greater than $5 \mathrm{~dB}$ are presented, i.e., $\sim 66 \%$ of the $\sim 850000$ projected signals. From the bottom to the top, the figures present plots of the time variability of the

1) omninoise;

2) omnipower;

3) maximum beam power achieved in a group of beams centered on the known source location;

4) raw beam wander, i.e., raw direction of maximum beam power received looking in the general direction of the 276- $\mathrm{Hz}$ signal source;

5) smoothed beam wander after removing low signal-tonoise data $(<5 \mathrm{~dB})$ and impulsive noise;

6) array gain;

7) temperature variability at a depth of $37 \mathrm{~m}$ near the horizontal receiving array;

8) temperature variability at a depth of $57 \mathrm{~m}$ near the 300-Hz source;

9) time variability of the spatial coherence between horizontal array hydrophones 1 and 5, 1 and 10,1 and 15, 1 and 20, 1 and 25, and 1 and 29.

As noted earlier, the acoustic array was positioned so that no acoustic sources would be in the endfire beam. As a result, the spatial coherence of the acoustic signals presented here is not the transverse coherence. The omninoise (Figs. 5, 6, and 10) was extracted from the data during the 2-s source offtime interval (acoustic source projected a signal with a 50\% duty cycle, $2 \mathrm{~s}$ on and $2 \mathrm{~s}$ off). Both the omninoise and omnipower are the sum of the signal power received on 29 hydrophones. The $y$-axis scale is in decibels for the omnipower, omninoise, and beam-power plots.

Figs. 5 and 6 have a number of red rectangular boxes with tops rising above the $y$-axis,see $y=300$. The boxes enclose the time intervals during which array navigation is considered to be good. The array-gain plot [see Fig. 5(f)] shows minima during the time intervals outside the boxes, i.e., time intervals during which the array navigation was poor or not even attempted. These time intervals usually correspond to the onset of high-frequency temperature fluctuations at the horizontal array see $[\mathrm{Fig} .5(\mathrm{~g})]$. The high-frequency temperature fluctuations were caused by the passage of internal wave packets. The internal wave packets had large currents associated with them [6] and caused the horizontal array to be physically displaced during their passage. Neither the long baseline pulsed navigation system nor the navigation procedures outlined in Appendix I could reliably navigate the horizontal array during these strong current events.

The 24-h period JD 125-126 is part of the longest time interval during which the horizontal array was continuously navigated. This section of data (Fig. 10) will be the focus of the following discussions. As can be seen by looking at the temperature records, this time interval is during a lull in the arrival of the large-amplitude high-frequency internal wave packets. Conclusions drawn concerning beamformer response should thus be viewed as the most optimistic performance that can be expected at the experiment site during this time of year. Due to the abovementioned array motion, we do not have beamformed data sets with enough temporal continuity to unequivocally draw conclusions concerning the limits that the higher energy environmental places on acoustic array performance. Future work may still provide some estimates.

\section{A. Omninoise}

The array omninoise power is displayed for all time intervals when the omnipower to omninoise SNR was greater than $5 \mathrm{~dB}$. Blank spaces in the data occur when the signal to noise ration was $<5 \mathrm{~dB}$. The most striking feature of the omninoise signal is that the increase in noise level is periodic and correlated to the arrival of high-frequency internal waves at the receiving array [see Figs. 5, 6, and 10(a) and (g)]. During these time intervals, current induced array motion and sand and gravel movement contributed to increased noise levels.

\section{B. Omnipower Fluctuations}

A quick look at Figs. 5, 6 and 10 reveals that the

- omnipower level was quite variable, with signal fluctuations of $15 \mathrm{~dB}$ or larger;

- omnipower signal intensity changes of $15 \mathrm{~dB}$ occurred over time intervals as short as $7 \mathrm{~min}$;

- on time scales as short as $20 \mathrm{~s}$, the omnipower signal level fluctuates by as much as $4-6 \mathrm{~dB}$;

- omnipower signal fluctuations were larger and had higher frequency variability during the JD 127-135 time when the larger amplitude high-frequency internal waves were present in the acoustic propagation path.

Histograms of the omnipower fluctuations (Fig. 8) broken into four 4-d blocks provide a quantitative measure of the omnipower signal fluctuations. Due to the rejection of signals with signal to noise less than $5 \mathrm{~dB}$, the total signal count in each 4-d time block is not the same. From a qualitative standpoint, there does not seem to be a striking differences between the histogram shapes. By visually integrating the area under the histogram, the reader can estimate the amount of time the fluctuations are between $\pm 3 \mathrm{~dB}$ and so forth. Large amplitude fluctuations, for instance $> \pm 5 \mathrm{~dB}$ occur $>10 \%$ of the time. The histogram does not show whether the larger amplitude fluctuations about the mean occur together or separately. A study of the time series seen in Figs. 5, 6, and 10 indicates that the large-amplitude fluctuations are often time correlated.

The frequency content of the omnipower for an 8-d section of the data is shown in Fig. 7(b1). The power spectrum for two 4-d subsections (JD 122.65-126.3 and JD 126.3-129.9) of the array omnipower is plotted in Fig. 7(b2) and (b3). In the JD 126.3-129.9 time intervals, high-frequency internal waves were perturbing the temperature field [Fig. 7(a3) and Fig. 7(c3)] at both the source and receiver sites. In the latter time interval, when the higher frequency internal waves fields were present in the acoustic propagation path, both the omnipower and temperature fluctuations at the receiving array and the source locations had higher energy in the short-period portion of the spectrum. This is to be compared to the period when the high-frequency omnipower fluctuations were smaller in amplitude. The spectra from the two subsections of 


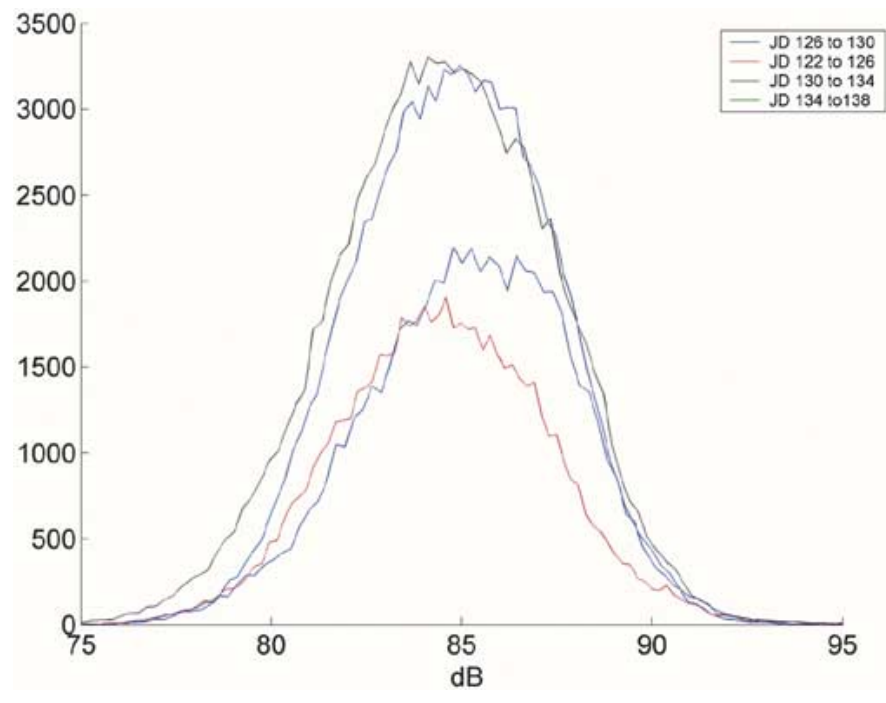

Fig. 8. Histogram of omnipower variability in four 4-day time intervals.

the omnipower show that the fluctuation were nonstationary. The high-frequency fluctuations of the omnipower increase with increasing temperature fluctuations at the source and receiver locations.

\section{Horizontal Array Performance-Conventional Beamformer}

1) HLA Geometry: The ASIAEX South China Experiment was originally designed to have two horizontal arrays oriented orthogonally to each other, one with a broadside beam pointed downslope and the other with a broadside beam pointed along a shelfbreak isobath. Due to equipment difficulties, only one array was deployed. In an effort to meet the experimental objectives, the deployed horizontal array was placed so that no acoustic sources were in the array's endfire beams.

Although the horizontal array used during the experiment was negatively buoyant in water, the currents associated with tidal flow and the passage of the Luzon Basin internal wave packets displaced the array at least one to two times per day.

In order to effectively apply a conventional narrow-band beamformer to the horizontal array data, the relative positions of the hydrophones have to be known to within a small fraction $(\sim 1 / 10 \lambda)$ of an acoustic wavelength. Due to the fact that a medium frequency $(\sim 10 \mathrm{kHz})$ long baseline pulsed acoustic navigation system placed at the experiment site was not reliable, because of the internal wave perturbations of the sound-speed field, a new array navigation methodology that utilized the phase front of the moored acoustic signals was developed by S. Wolf (see Appendix I). This technique was used repeatedly to navigate the position of each hydrophone in the horizontal array for the duration of the experiment. The time-variable hydrophone locations were repeatedly updated in the conventional beamformer. Changes in the horizontal array geometry for the duration of the experiment are plotted in Fig. 9. The array was often displaced several tens of meters approximately every $12 \mathrm{~h}$, sometimes every $6 \mathrm{~h}$. The phase-front navigation required long integration times, i.e., long periods of time when the horizontal array was stationary. It was not applicable to those time periods

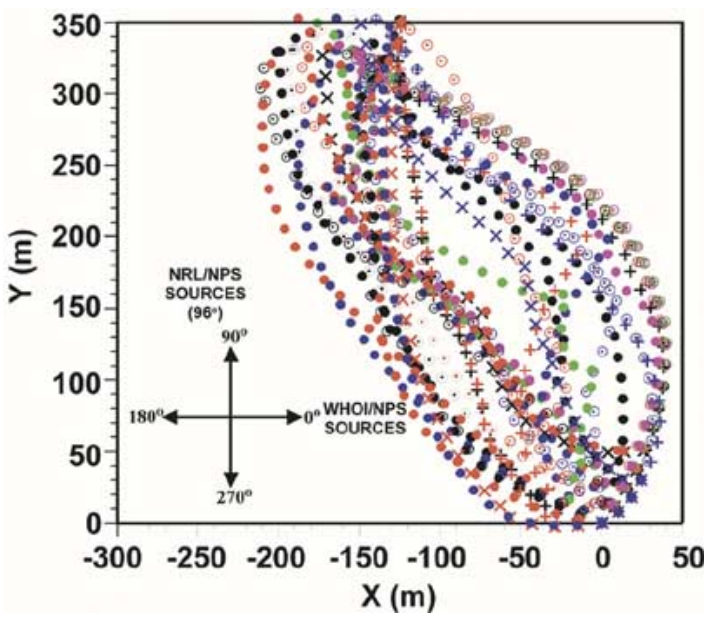

Fig. 9. Horizontal array shape versus time. The 32 hydrophone locations for each time interval during which the array remained stationary are plotted with a common symbol, e.g., + , o.

during which the array was in motion. Consequently, there are time intervals during which the horizontal array element's positions were not known and reliable beamforming could not be accomplished. Unfortunately, these time intervals often are the periods of time during which the environment was most dynamic. As mentioned earlier, the red blocks in Fig. 5 outline the time intervals during which good array navigation was available.

2) Horizontal Array-Conventional Beamforming: A 276$\mathrm{Hz}$ narrow-band component of the acoustic signals received from the $300-\mathrm{Hz}$ moored broad-band acoustic source (i.e., a single Fourier transform line) was processed using a conventional linear cross-spectral density matrix based beamformer. Cross-spectral density matrices were integrated over $4 \mathrm{~s}$. The beamformer used 29 contiguous array elements. The entire 16-d data set was beamformed. Numerical simulations using the measured array element locations indicate that the $-3 \mathrm{~dB}$ beamwidth of this array for a plane wave field at the $300-\mathrm{Hz}$ source bearing is about $2^{\circ}$.

As mentioned before, the horizontal hydrophones were spaced uniformly at an interval of $15 \mathrm{~m}$. Consequently, the array sparsely sampled the $276-\mathrm{Hz}$ acoustic field. Beam processing of the acoustic field with such a sparse array (if it were straight) would yield an aliased beam pattern, with grating lobes of equal intensity to the beam-power maximum in the source's true direction. In this experiment, the periodic symmetry inherent in the beam-steering coordinate of the linear, sparse array was broken by its two-dimensional (2-D; planar) shape, see Fig. 9. As a result, the phase alignment of the signals at all the elements, the condition that produces maximum beam power, is achieved only in a single steering direction and the source is unambiguously located. Beamformer output will be presented for the maximum received signal in beams pointed in the general direction of the NRL 300-Hz source.

3) Impact of Wavefront Curvature: The near-field range ( $L^{2} / \lambda$ where $L$ is the array length and $\lambda$ is the acoustic wavelength) of the $465-\mathrm{m}$ array oriented with the $300-\mathrm{Hz}$ source at array broadside is approximately $43 \mathrm{~km}$. This is greater than 


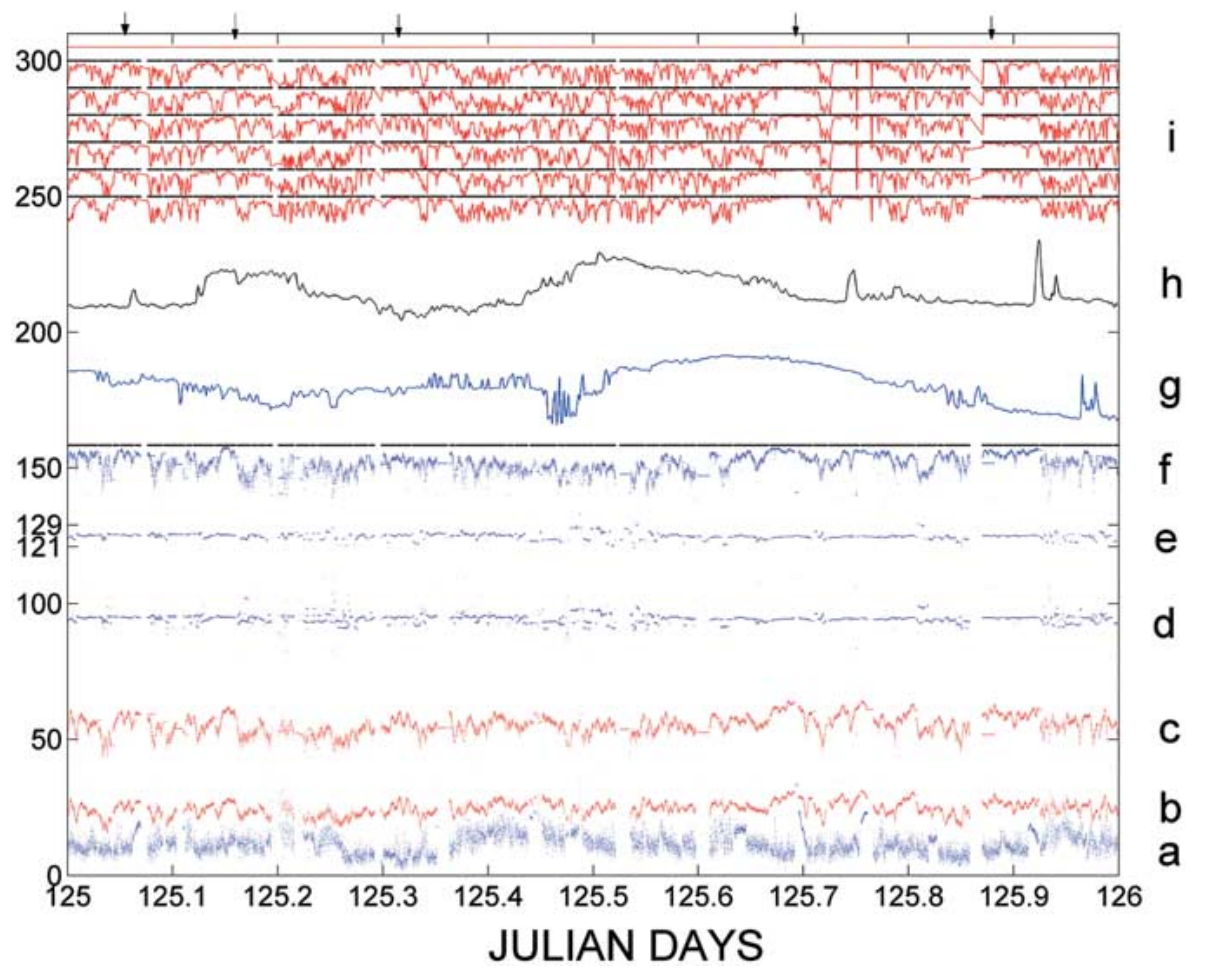

Fig. 10. Twenty-four-hour (JD 125-126) narrow-band 276-Hz array response and temperature record composite. (a) Omninoise, (b) omnipower, (c) beam power, (d) raw direction to $276-\mathrm{Hz}$ acoustic source, (e) direction to acoustic source with noise removed, (f) array gain, (g) 37-m depth temperature record at the vertical line array, (h) 57-m depth temperature record near the sources, and (i) temporal variability of the spatial coherence for horizontal array phones 1 and 5, 1 and 10, 1 and 15, 1 and 20,1 and 25, and 1 and 29, bottom to top, respectively. The $y$-axis has decibel units when referring to omnipower, omninoise, and beam power. In the case of array gain, four units of $\mathrm{dB}=1 \mathrm{~dB}$ of array gain. Absolute temperature scale is presented in Fig. 12(a) and (b). The direction to acoustic sources with noise removed [Fig. 10(e)], 121-129 represents (four degrees). In the coherence plots (i), ten units of the $y$-axis represents a change in coherence from 0 to 1.

two times the actual range of the source used in this experiment. Even so, wave front curvature corrections in the conventional beam former were not implemented for two reasons. First, the array navigation procedure (see Appendix I) used the mean acoustic signal phase differences. As a result, the beam-steering corrections in the source's direction compensated for the acoustic field wave front curvature. Second, for beams pointed away from the sources, numerical simulations indicated that wave front curvature has minimal impact on the beamformer. For the actual array shape, the array signal gain and 3-dB beamwidth for all combinations of cylindrical and plane wave acoustic fields and steering vectors were calculated. For these cases, the differences in array signal gain $(\sim 0.5 \mathrm{~dB})$ and beamformer bearing bias $\left(\sim 0.2^{\circ}\right)$ were considered small enough to let one ignore wavefront curvature corrections.

4) Beamformer Results: The time variability of the beam power, array gain, and beam wander for the maximum beam in the general direction of the NRL $300-\mathrm{Hz}$ source is plotted for the entire 16-d set (Fig. 5). The magnitude of the beam power is only meaningful in the time intervals for which good array navigation was available; i.e., as indicated previously within the red boxes.

a) Beam power: Within the good navigation boxes, beam-power intensity changes of $15 \mathrm{~dB}$ occurred over time intervals as short as $7 \mathrm{~min}$. On time scales as short as $20 \mathrm{~s}$, the beam-power levels fluctuated by as much as $4-6 \mathrm{~dB}$. Figs. 6 and 10, which present subsections of the full data set (Fig. 5)

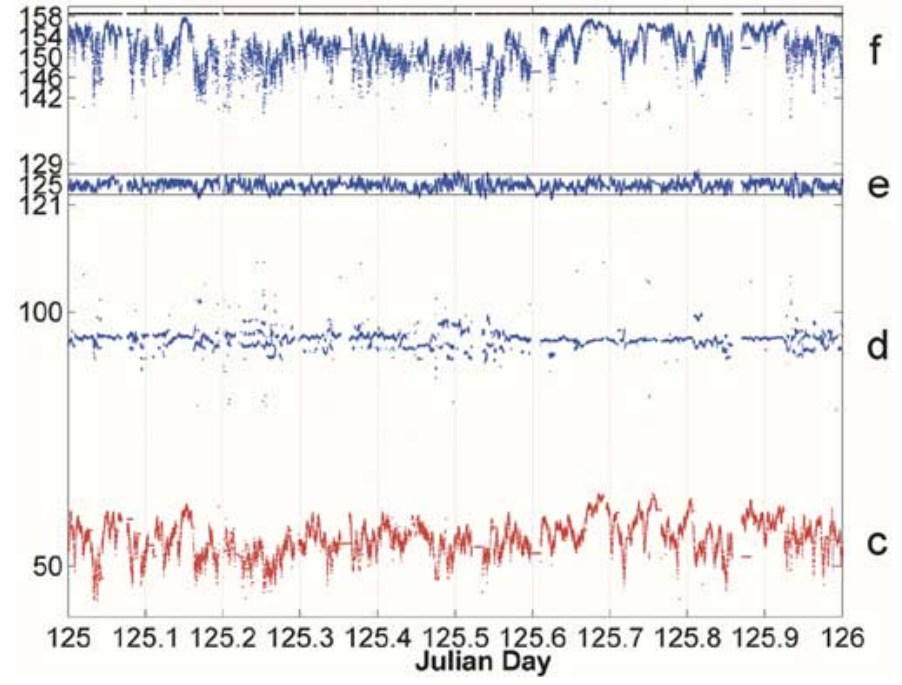

Fig. 11. Close up of Fig. 10 specific series. (c) Beam power, (d) raw direction to the $276-\mathrm{Hz}$ acoustic source, (e) smoothed direction to acoustic source with noise removed and 1-min running average, and (f) array gain. The $y$-axis has decibel units when referring to beam power. In the case of array gain, four units of $\mathrm{dB}=1 \mathrm{~dB}$ of array gain. Absolute temperature scale is presented in Fig. 12(a) and (b). The direction to acoustic sources with noise removed [Fig. 11(e)], 121-129 represents four degrees.

show that the beam-power variability mirrors the omnipower variability. As in the case of the omnipower, the changes in the beam-power amplitude and period appear to qualitatively follow the changes in the spectal character of the temperature 
field. Larger amplitude and shorter period fluctuations occur during the time intervals (Fig. 5) JD 128-135, when large amplitude and short-period internal waves were present. Note that the magnitude of the beam power falls off sharply in each of the time intervals that the array could not be navigated. This fall off indicates that the array navigation procedures developed by Wolf were improving our knowledge of the array element locations.

b) Array gain: Array gain has been calculated by dividing the beam power by the omnipower. The array gain $(\sim \times 4)$ for an array consisting of 29 phones is plotted in Figs. 5, 6, and 10 (see line $\mathrm{f}$ ). The horizontal line above the array-gain curves is the array gain anticipated from 29 phones, i.e., $10 \log (29)$. These figures provide a qualitative measure of the time variability of the array gain. The array gain is strongly correlated to changes in omnipower and omninoise. If omnipower is large and omninoise is small, i.e., good signal-to-noise characteristics, array gain approaches within $1 \mathrm{~dB}$ of the $10 \log (29)$ curve (see arrows at the top of Fig. 10). The coupling of omnipower and omninoise signal-to-noise variability and array-gain variability, along with the limited temporal continuity in the array element navigation, limits our ability to unequivocally relate array-gain variability to the fluid dynamic perturbation of the sound-speed field. Sections of the data are certainly suggestive that the correlation exists.

c) Beam wander: The maximum power beam wander is plotted in Figs. 5, 6, 10, and 11, line e. In Figs. 5, 6, and 10, line $\mathrm{e}$, the beam wander is plotted for all signals with $>5 \mathrm{~dB}$ signal to noise. All signals contaminated with impulsive noise were also removed. In Fig. 11, the beam wander is averaged over $1 \mathrm{~min}$. The magnitude of the beam wander is only meaningful in the sections of data with good array element navigation. Fig. 11 is a close up of the beam-wander plot for JD 125-126. Two-degree steps are marked on the $y$-axis. The 24-h section of data experiences a maximum beam wander of $\pm 2^{\circ}$. Within the resolution of the array and for data with a 1-min running average, there is no apparent coupling between environmental variability and beam-wander variability. Again, this is a period of time during which the internal wave energy was smaller than the spring tide phase of the lunar cycle.

d) Spatial coherence: The spatial coherence between horizontal array hydrophones 1 and 5, 1 and 10, 1 and 15, 1 and 20,1 and 25, and 1 and 29 was calculated in the frequency domain following a procedure from Bendat and Piersol [8]. The results $(\times 10)$ are plotted in Figs. 5, 6, and 10, line i, in ascending order. The horizontal black line above each time series represents a coherence of 1 . If the coherence were stable in time, each coherence plot would be a straight line. As can be seen in Fig. 5, the coherence for each hydrophone pair has significant temporal variability over the 16 -d time frame. The time intervals JD 122-126 and JD 136-138 show less amplitude variability, i.e., more white under the curves. In general, there is a qualitative correlation between the presence of high-frequency large-amplitude internal waves and high-frequency variability in the spatial correlation between hydrophones during the JD 126-136 time frame. The correlation is clearer in the 8-d time series (Fig. 6). The spatial coherence plot has not had the time intervals during which the array was in motion removed.
The 24-h record (Fig. 10, line i) has sufficient resolution to show the details of the temporal variability in the spatial coherence and can be compared to temperature, array gain, beam power, omnipower, and omninoise variability. During this time, the hydrophones are thought to be stationary in position. We note three points. First, the regions of highest array gain, mentioned before and marked with arrows on the top of the figure, are correlated in time with high interphone signal correlation. Second, the regions of high spatial correlation and large array gain are correlated with the regions of large beam power. Third, these regions of high correlation appear to be correlated with time intervals of relatively small temperature gradients at both the source and receiver locations. In regions of larger temperature fluctuations, the spatial coherence is significantly more variable for all hydrophone separations. During poorer correlation times, the SNR and array gain also have smaller values.

In summary, due to the multiparameter variability, the strongest statement that we can presently make is that the spatial coherence is temporally variable, the magnitude of the coherence is largest when the SNR on the array is highest and the temperature variability at the source and receiver is small. Since the array and source are both fixed, the coherence variability obviously has some correlation to internal wave-induced sound-speed variability.

A close up of the temperature time series at the source and receiver is provided in Fig. 12. Fig. 13 provides an overlay of the time series for the spatial coherence, temperature field, and array gain for array apertures of 5, 10, 15, 20, 25, and 29 hydrophones. When evaluating the interrelationship of temperature, coherence, and array gain, the reader must refer back to Figs. 5, 6, and 9 and relate the signal-to-noise variability to the array gain and coherence variability.

Fig. 13 shows that the array gain and spatial coherence were at a maximum when the temperature variability near the source and receiver arrays was small. Conversely, when the temperature fluctuations were the largest, there was a decrease in the spatial coherence magnitude and an increase in its temporal variability. There are exceptions. Deep fades in omnipower, beampower, and array gain occur when temperature variability at either end of the acoustic propagation path was nominal. Since the source and receivers were stationary, the fades should be related to sound-speed field variability. As mentioned earlier, internal wave packets could occupy the acoustic propagation path and not be detected at either of the acoustic source or receiver positions. In order to positively identify the physical cause of the signal fades, this experiment needed higher spatial resolution temperature and salinity sampling along the acoustic propagation path.

e) Array-gain variability as a function of aperture size: The array gain is plotted for JD 125 for $5,10,15,20,25$, and 29 phone apertures (n-1 separations) in Figs. 13 and 14. The aperture size in wavelengths for the $276-\mathrm{Hz}$ signal is listed in Table I. The aperture size does not increase linearly with the number of phones, because the hydrophones at the end of the array numbers 20,25, and 29 are oriented so their distance from the acoustic source is nearly constant, i.e., around 29 lambda. The horizontal black line above each curve is $10 \log (N)$ where $N$ is the number of phones. 

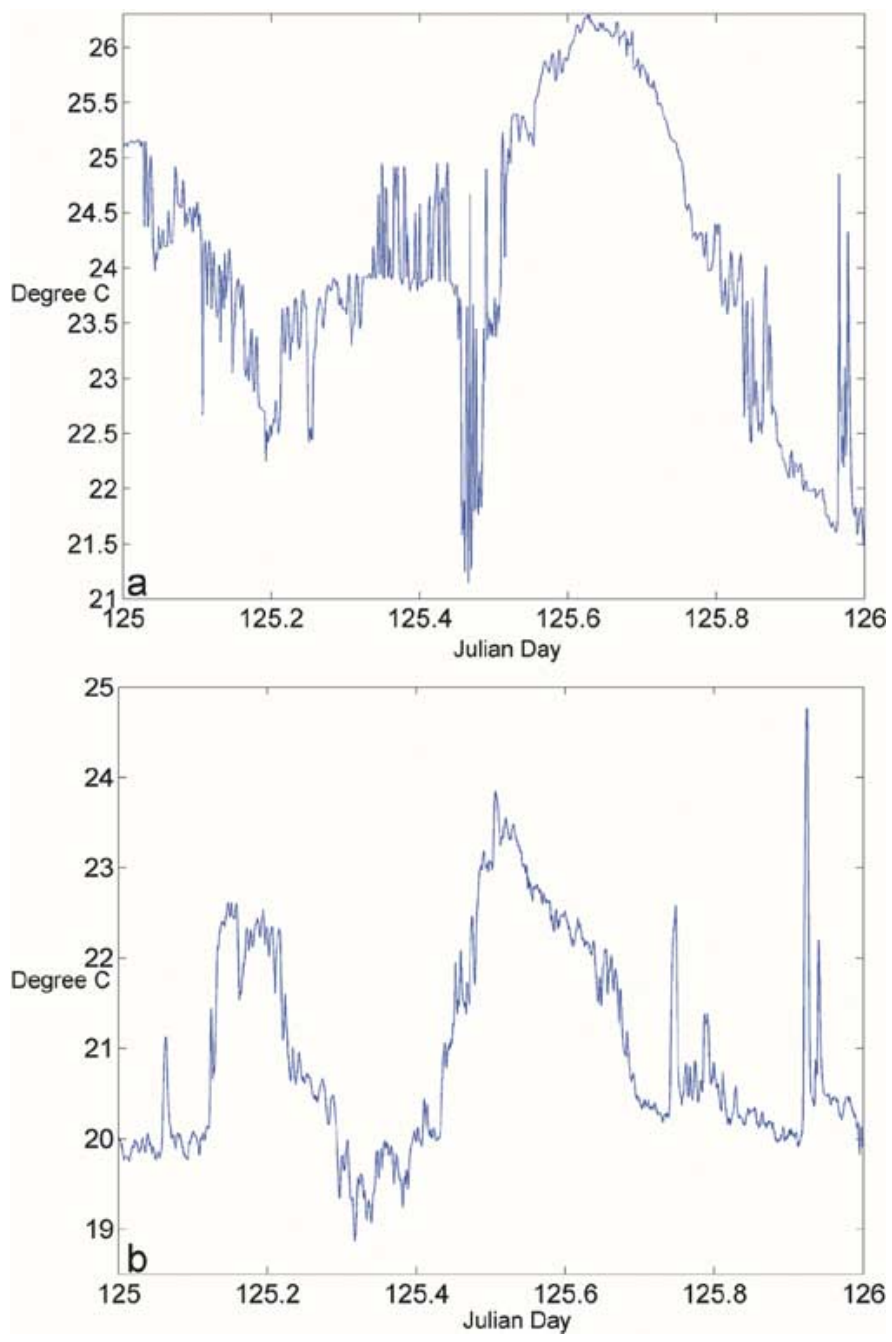

Fig. 12. Close up of the temperature record in Fig. 10. When depression internal waves are propagating past the thermistor, the temperature rises. The sudden drop in temperature at JD 125.45 may be related to the VLA being depressed in depth due to increased currents. Time intervals during which spatial coherence is maximum in Fig. 10 correlates with time intervals during which temperature variability at the receiving array is at a minimum. Deep fades in beam power sometimes occur, even though the temperature at the source and receiver are fairly constant.

The primary conclusions that are drawn from the figures are

1) the shorter the array, the closer the array gain approaches $10 \log (N)$;

2) the array gain is time dependent;

3) the array gain is best in high signal-to-noise environments and during time intervals in which the temperature variability at the source and receiver is small;

4) there are deep fades in array gain that appear to be unrelated to temperature variability at either the source or receiver locations.

Array gain was poor for all array lengths when internal waves were propagating past the receiving array (e.g., JD 125.3-125.55). During this time interval, there was an abnormal decrease in temperature. This decrease suggests that high currents associated with the internal waves are depressing the vertical line array and the attached temperature sensor. During this time, there also is a decrease in SNR on the horizontal array hydrophones. There were no current meters at the
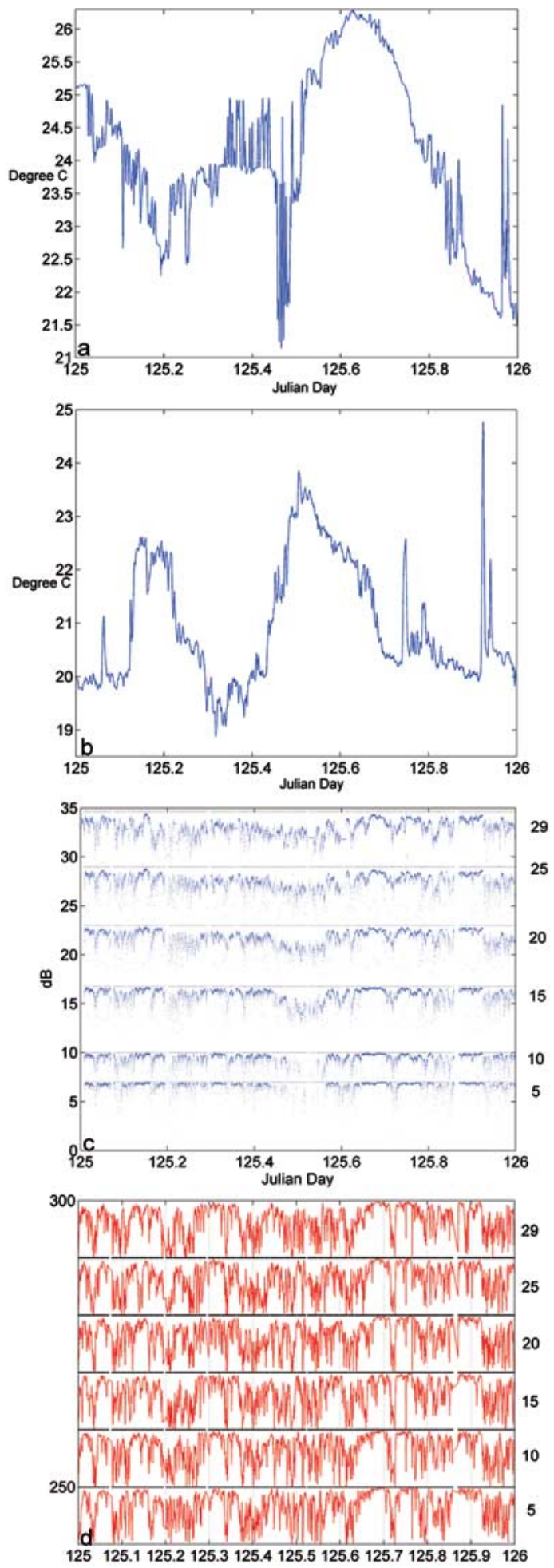

Fig. 13. (a) Temperature variability at acoustic receiver for a 24-h time interval. (b) Temperature variability at the acoustic source for a 24-h time interval. (c) Array gain versus number of phones. (d) Spatial coherence versus phone separation.

receiver location to corroborate the inference of large currents. A separate sudden decrease in array gain and spatial coherence occurs in the JD 125.93-126 time interval and is correlated with the arrival of internal waves at the source location. There also is a corresponding decrease in signal to noise due to the presence of a research vessel in the vicinity of the receiving 


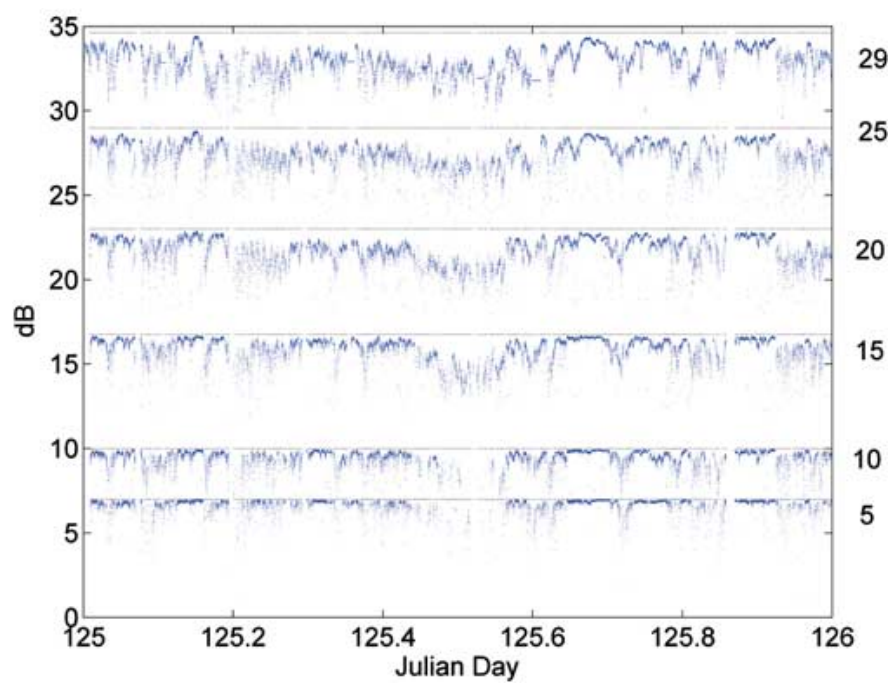

Fig. 14. Array gain in decibels versus time and number of phones.

TABLE I

ARRAY APERTURE SIZE IN WAVELENGTHS FOR THE 276-Hz SigNAL

\begin{tabular}{c|c}
\hline Number of Phones & Array length in Lambda \\
\hline & \\
\hline 5 & 3.26 \\
\hline 10 & 11.06 \\
\hline 15 & 19.59 \\
\hline 20 & 28.65 \\
\hline 25 & 29.51 \\
\hline 29 & 29.58 \\
\hline
\end{tabular}

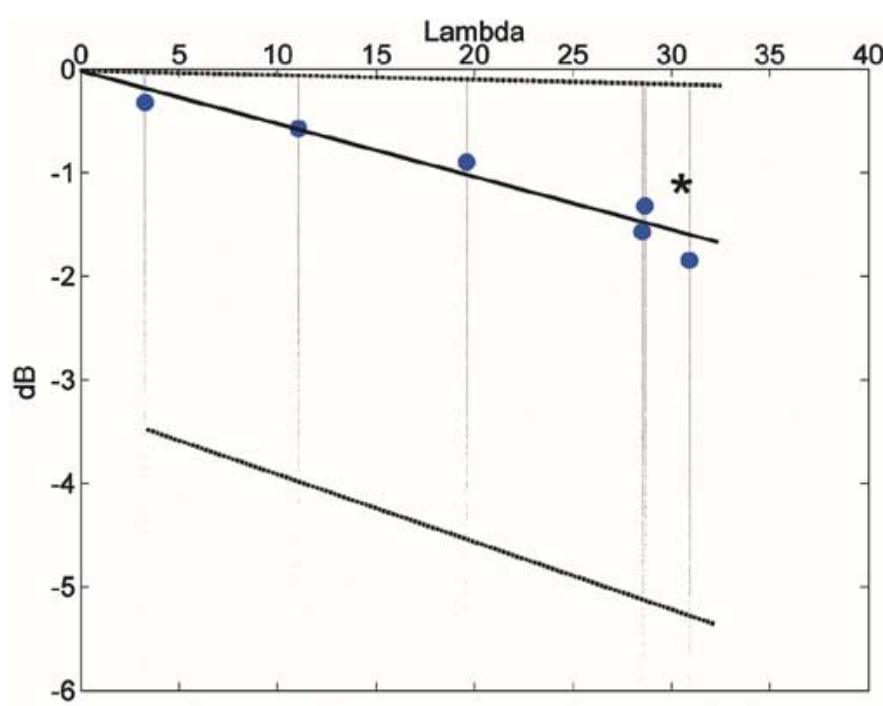

Fig. 15. Composite plot of array-gain variability versus aperture length in wavelength. Measurement by Carey et al. [9] in the Straits of Korea is denoted by $(*)$.

array. Both of these sections of data are supportive of a correlation between sound-speed variability and array-gain variability. The data is not as definitive as desired because of the constantly changing SNRs.

f) Variability in Array-Gain Degradation: The array-gain degradation for each of the above aperture lengths (Fig. 15) varies during the 24-h time period. The smallest array-gain degradation ranges from near $0 \mathrm{~dB}$ for the short apertures

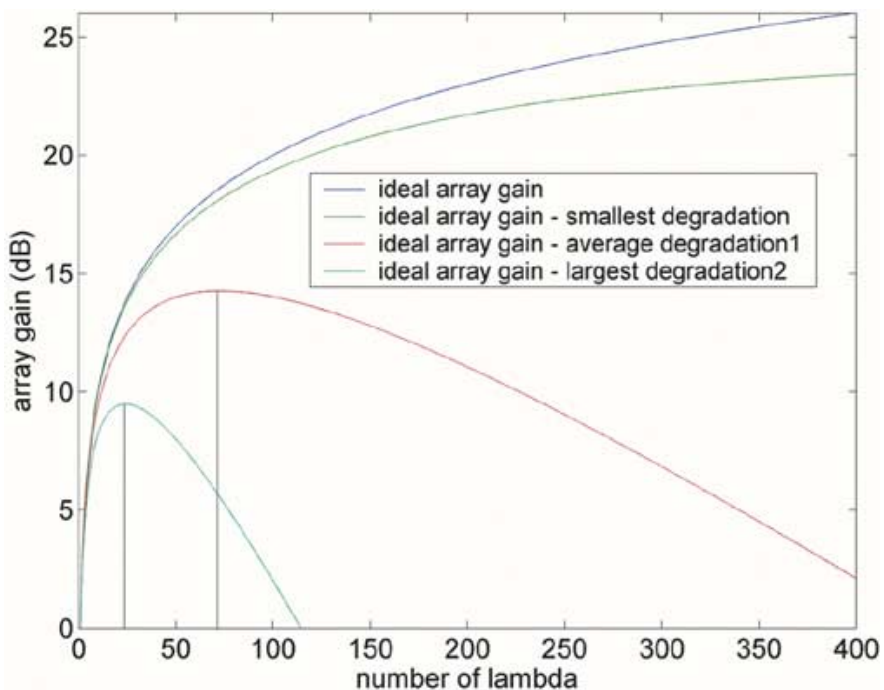

Fig. 16. Realizable array gain for best, average, and worst array-gain degradation measured during the 24-h time segment.

$(3.2 \lambda)$ to $\sim-0.2 \mathrm{~dB}$ for the longer aperture $(\sim 30 \lambda)$. The greatest array-gain degradation ranged from about $-3.5 \mathrm{~dB}$ for the short apertures to $-5.5 \mathrm{~dB}$ for the longer apertures $(\sim 30 \lambda)$.

The average array-gain degradation for each aperture during the 24-h time period is noted with a point for each aperture length. The straight line through each of the points has a slope of $-0.06 \mathrm{~dB} /$ wavelength for the $276-\mathrm{Hz}$ signal source at a range of $18.9 \mathrm{~km}$. The slopes for the best case (upper dashed and dotted curve) and worst case (lower dotted curve) are -0.00647 and $-0.18 \mathrm{~dB} /$ wavelength, respectively. The asterisk is the arraygain degradation estimated for the Straits of Korea by Carey $e t$ al. [9] for a similar acoustic wavelength and range. The averaged array-gain degradation observed in the South China Sea and the Carey measurement are comparable in magnitude. The slopes can be used to estimate the size of an aperture that will realize ideal array gain in the propagation conditions experienced during the 24-h time period. Realizable array gain versus aperture size in wavelength is plotted (Fig. 16) for the best, average, and worst array-gain degradations measured during the 24-h time period. The plot indicates that array lengths $>400 \lambda$ and $\sim 70$ and $25 \lambda$ will realize array gain for the best, average, and worst cast array-gain degradations measured. Following Carey [9], the measured array-gain degradation can be used to estimate coherence lengths. For an exponential spatial coherence function raised to the $n=1.5$ power, the spatial coherence works out to 85,30 , and 5.5 lambda for a $\sim 30-\lambda$ array.

The $276-\mathrm{Hz}$ signal was generated more than 43000 times during the 24-h time period. A histogram of the variability of the array-gain degradation for each aperture size provides a measure of the distribution of array-gain degradation versus aperture size (Fig. 17). As the aperture length increases, the array-gain degradation probability function broadens. The Carey measurement mentioned before is plotted for the 29 lambda aperture length, i.e., the asterisk. The asterisk location is less than but close to the average array-gain degradation measured during this experiment. The histogram gives a measure of the frequency of occurrence of array-gain degradation above and below the calculated average value for this 24 -h period. 

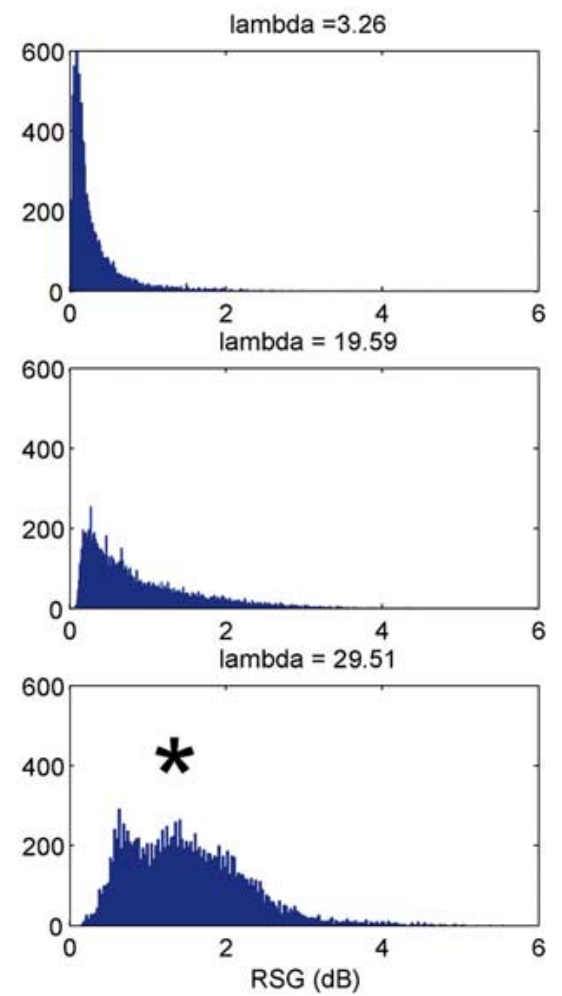
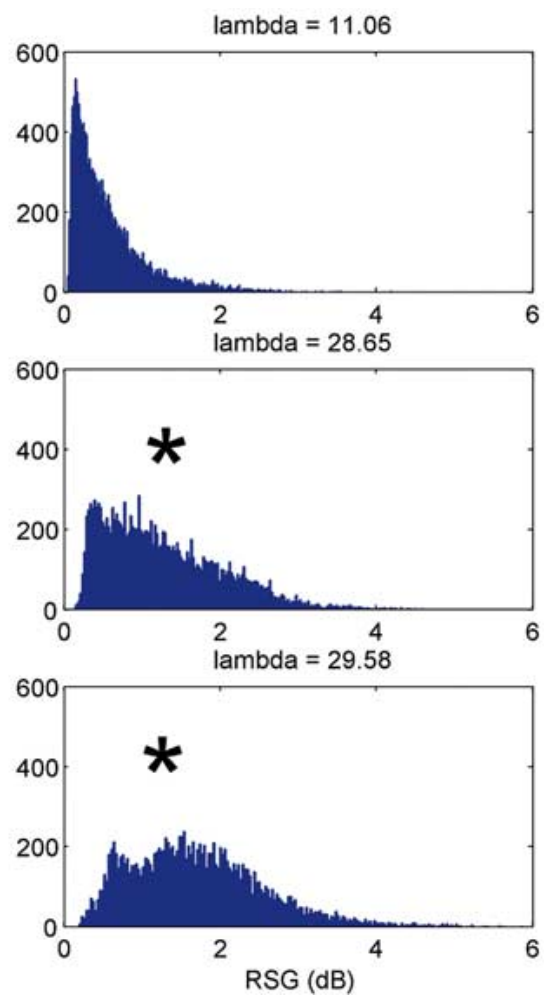

Fig. 17. Histogram of the array-gain degradation measured over a 24-h time period. The Carey et al. [9] measurement for the Straits of Korea is denoted by the asterisk above the $\sim 29$ lambda length apertures.

\section{CONCLUSION}

The ASIAEX South China Sea experiment, which was performed in the vicinity of the continental shelf break, has connected the internal tide and internal wave perturbation of the sound-speed field to the temporal variability in the performance of a bottomed horizontal array. Results for a $276-\mathrm{Hz}$ acoustic signal propagating along the shelf break for a distance of $\sim 19 \mathrm{~km}$ are summarized.

The spectral composition of both the array's omnipower output and the temperature field at the source and receiver locations was nonstationary in the high-frequency bands (periods between $\sim 0.1$ to $0.01 \mathrm{~min}$ ). There was a strong correlation between omnipower and beampower high-frequency variability and temperature/sound field variability induced by internal waves. The nonstationarity is related in part to the spring to neap lunar cycle induced changes in the characteristics of the internal tide and associated nonlinear internal wave fields. Omnipower fluctuation variability was $> \pm 5 \mathrm{~dB}$.

Horizontal array gain was also temporally variable. The variability was correlated to the presence of internal waves in the acoustic propagation path. Array-gain degradation was both array length and time dependent. Changes in array gain were strongly correlated with changes in spatial coherence across the array.

In this experiment, the horizontal array and source were moored and acoustic signals were projected every $4 \mathrm{~s}$. As a result, histograms of the array-gain degradation as a function of array aperture length were generated. The histograms set a lower bound on the impact of sound-speed field variability on array-gain variability. These histograms will be used to develop array-gain probability-distribution functions. They imply that the array-gain degradation distribution function will change as array length increases. As an illustration, the array gain degradation for a $29 \lambda$ array over a 24 -h period ranged from $\sim-15$ to $>-5.5 \mathrm{~dB}$ with an average value of $\sim 1.5 \mathrm{~dB}$ while an $11 \lambda$ array's degradation ranged from $\sim 0.1$ to $\sim 4 \mathrm{~dB}$ with an average value of $\sim 0.6 \mathrm{~dB}$. The shorter arrays had a much tighter clustering (Fig. 17) of the array gain degradation than the longer aperture. Following Carey [9], the coherence lengths of the signals received by the $29 \lambda$ array for the smallest, average, and largest array-gain degradation were calculated to be about 85 , 30 , and $5.5 \lambda$. The coherence length for the average array gain degradation is similar to the $\sim 30 \lambda$ estimates made by Carey [9]. The histograms expand our knowledge concerning the impact of internal tide and internal wave induced sound-speed fluctuations on array-gain degradation variability. Aperture lengths for maximum realizable gain for the smallest, average, and maximum array gain degradation measured with the $29 \lambda$ array were estimated to be $>400, \sim 70$, and $\sim 25 \lambda$, respectively.

\section{APPENDIX \\ ARRAY ELEMENT LOCALIZATION}

The acoustic receiving array was laid so that it was not endfire to either the cross-slope or downslope acoustic sources. As a result, the phase fronts from both downslope and cross-slope sources could be used to localize the individual hydrophones in the array.

The angle formed at the receiving array by lines drawn to the cross-slope and downslope acoustic source mooring positions is about $95^{\circ}$. Since the source bearings are not co-incident, element-to-element cross-correlation analysis of the acoustic signals from these sources can be used to localize the array ele- 


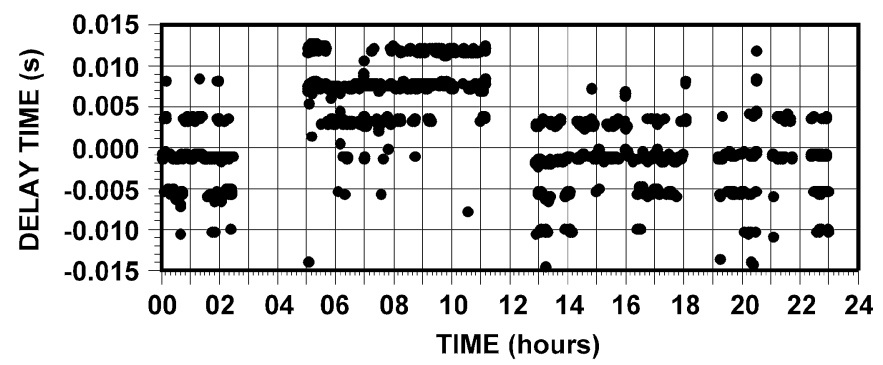

Fig. 18. Delay time of the largest positive peak of the cross-correlation function between HLA channels 1 and 2 for each individual 224-Hz PRN pulse over a 24-h period (JD 123). Gaps in time are due to poor signal-to-noise conditions.

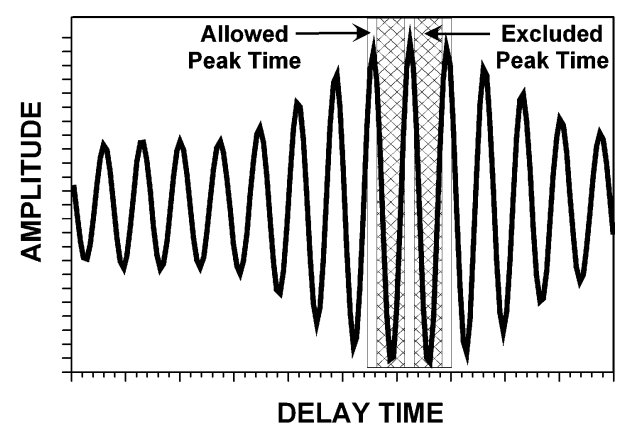

Fig. 19. Sketch of a nominal cross-correlation function between array element pairs, illustrating the clustering of delay times seen in Fig. 17. Small amplitude variations between pulses will produce a peak value at any of the allowed delay times.

ments in the two horizontal dimensions with respect to an arbitrary reference element. The elements can be localized with accuracy sufficient to support phase coherent processing of the acoustic signals whenever the array was stationary for periods on the order of a few hours.

The time-domain cross-correlation between acoustic signals received on all horizontal array element pairs was determined by using standard techniques. A typical result for the $224-\mathrm{Hz}$ (PRN) signal cross-correlation processing between HLA channels 1 and 2 is shown in Fig. 18. Similar processing for all HLA hydrophone pairs was applied to every 224-, 300-, and $500-\mathrm{Hz}$ signal pulse recorded over the 16-d experiment. Crosscorrelation results with poor signal to noise were discarded. In the data selected for localization analysis, the peak of the cross-correlation function was typically $15-20 \mathrm{~dB}$ above the noise correlation.

During short intervals, the peak 224-Hz cross-correlation delays are clustered about several discrete values at nominal 4-ms intervals (for example, the clusters about $-2 \mathrm{~ms}$ seen during 00-02 h in Fig. 18. The occurrence of discrete values of the maximum time delay, with intermediate gaps, can be explained by considering the typical cross-correlation amplitude trace shown as a solid line in Fig. 19. If the cross-correlation function were completely stable and noise free, the largest correlation peak would always occur at the delay associated with the peak of the central (boxed) maximum. If a small amount of noise is introduced, the correlation peaks may occasionally occur at times associated with nearby peaks (also clear boxed), but will not occur during the intervening times (cross-hatched peaks) unless the noise grows to become nearly as strong as the signal. The satellite peaks in the slightly noisy cross-correlation function

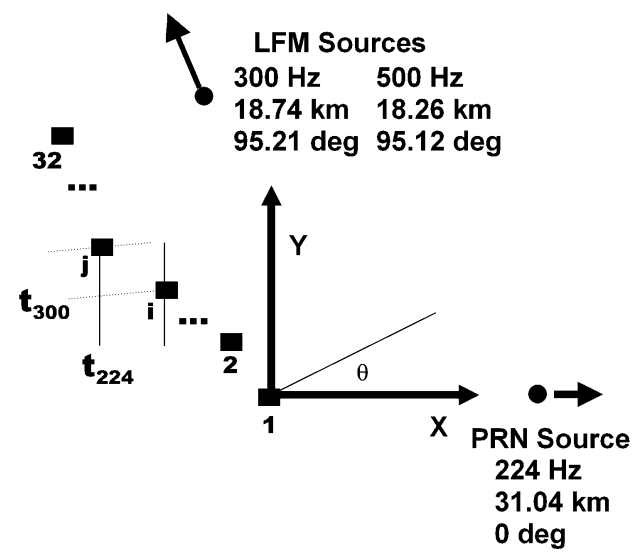

Fig. 20. Coordinate system used in horizontal array element localization. The $x$-axis points toward the 224-Hz PRN acoustic source. HLA channel 1 is located at $(0,0)$.

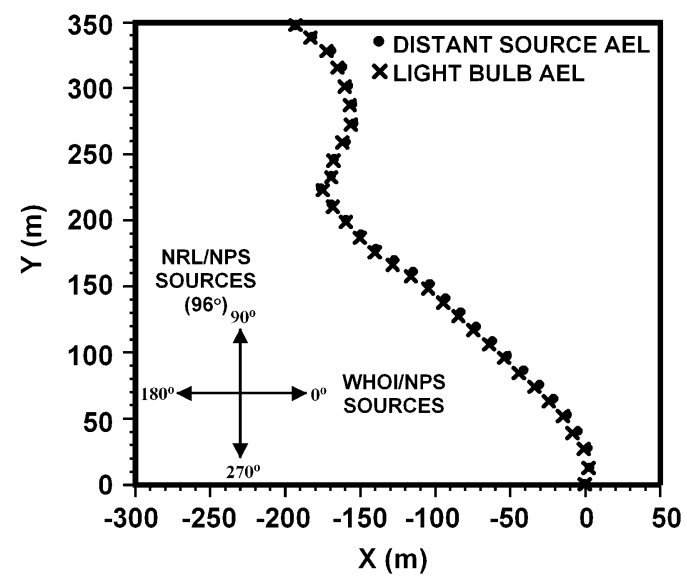

Fig. 21. Comparison of array element locations derived from light bulb implosion data and 300-Hz distant source data collected on JD 125.

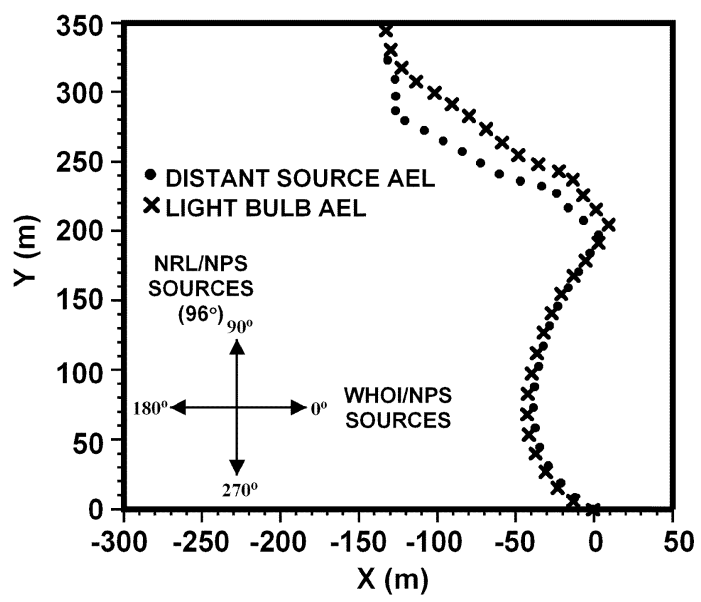

Fig. 22. Comparison of array element locations derived from light bulb implosion data and 300-Hz distant source data collected on JD 135.

will occur at intervals that are multiples of the reciprocal of the acoustic signal's center frequency, as seen in Fig. 19. Correlation plots for the other broad-band signals analyzed (300- and $500-\mathrm{Hz}$, not shown here) also showed delay time gaps between observed clusters of peak correlation corresponding to their respective signal periods. For each of the three source signals, the correlation delay between pairs of receivers was estimated by identifying the nominal time of the most frequently occurring 

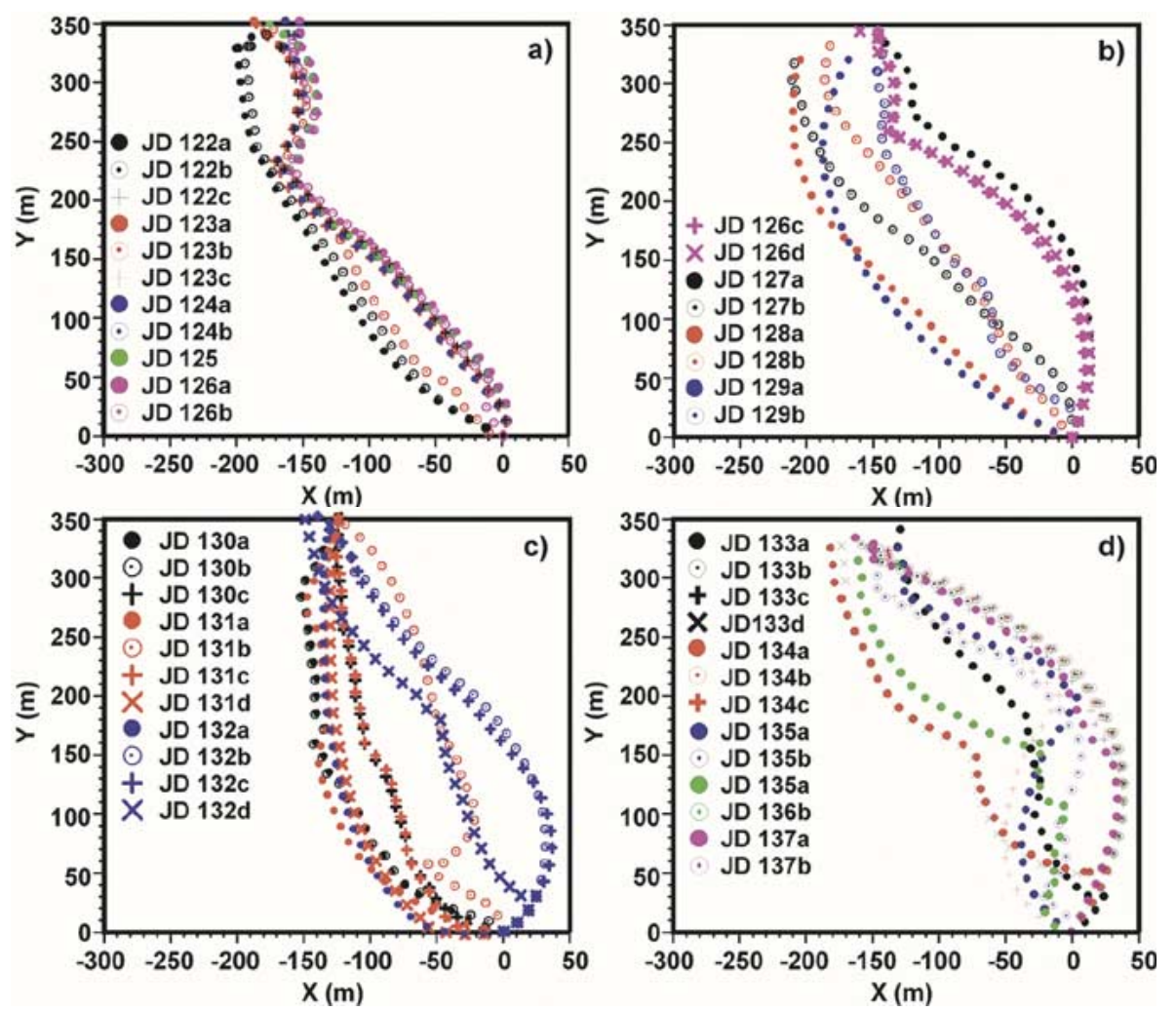

Fig. 23. (a) Array element locations for JD 122-127 representing a relatively stable period for array element positions. (b) Array element locations for JD 127-130. (c) Array element locations for JD 130-133. (d). Array element locations for JD 133-138.

delay and averaging all the delay values found within 0.4 periods of the nominal delay. Delay values on the "satellite" traces (separated by multiples of the acoustic period) were not used. Only the positive peak values (rather than the peak absolute value) was used to determine the interelement spacing, since the spacing will be used to correct beamformer phase shifts necessary to align phases of signals incident from the beamformer's steering direction.

The averaging interval was selected to include those times for which the delay appeared to be constant. If the delay suddenly shifted (as at 5 and $13 \mathrm{~h}$ in Fig. 18), the array was considered to have moved and new intervals were defined for use in a different localization. It was found that the periods of constant delay and the times of shifted values were the same for the three broad-band signals (224-, 300-, and 500-Hz), consistent with the assumption of array motion. During selected times associated with array motion, bumping and scraping noises could be heard that were not present during the intervals when the array appeared to be stationary.

We found that when we correlated adjacent hydrophone channels (1 and 2, 2 and 3, etc.) it was not difficult to identify the most prominent cluster of delay values. As the separation between array elements increased, we continued to observe the grouping of the delays into clusters in delay time, but it became difficult to identify the "correct" cluster. This difficulty is apparently the result of a weakening signal correlation as receiver separation increases. Since the prominent clusters in delay were readily identified with nearest neighbors, the array shape determination was obtained from the average correlations of adjacent element pairs.
The procedure for determining the array shape is indicated in Fig. 20. We arbitrarily use array element 1 , most distant from the vertical hydrophone array, as the origin of the coordinate system. The $X$-axis (and, therefore, array-steering angle $0^{\circ}$ in the beam processing in Section III is defined to pass through the 224-Hz PRN source location. The $Y$-axis is chosen normal to the $X$-axis, so the 300 - and 500-Hz LFM sources lie at about $95^{\circ}$ bearing. In this coordinate system, True North lies at about $162^{\circ}$. The $X$-value of element 2 is determined from the 224-Hz signal cross-correlation delay. The $Y$-value of element 2 is determined primarily from the $300-\mathrm{Hz}$ (or $500-\mathrm{Hz}$ ) signal cross-correlation delay, with a small correction for the value of $X$. The $X$ and $Y$ interval between elements 2 and 3 are similarly determined, with a small correction to $X$ for the value of $Y$ appearing as the temporary reference element moves off the $X$-axis. The location of an arbitrary element with respect to element 1 is determined by adding the $X$ and $Y$ increments. Since the 300and $500-\mathrm{Hz}$ signal cross-correlation delays primarily determine the $Y$-values, an estimate of the accuracy of the method may be obtained by comparing the $Y$-coordinates of the array elements independently determined at these two frequencies. It was found that the elements at the end of the array nearest the VLA (and most distant from the reference element 1) had typical $Y$-value differences (error accumulated as we integrate $Y$ over the entire array) of less than $1 \mathrm{~m}$ and often less than $0.5 \mathrm{~m}$. We note here that array element number 30 was unusually noisy for reasons not yet determined. One result of this high noise value is that it was often impossible to obtain a localization of this element. Consequently, we used only the 29 contiguous well-localized elements numbered 1 to 29 for the beam processing in Section III. 
TABLE II

ACOUSTIC ElEMENT LocAlization InTEgration Times. ARray Motion And Acoustic Noise Were Minimal During These Periods

\begin{tabular}{l|r|r}
\hline \hline IDENTIFIER & START & END \\
\hline \hline $122 \mathrm{a}$ & Julian Day) & \multicolumn{1}{l}{ (Julian Day) } \\
\hline $122 \mathrm{a}$ & 122.4 & 122.52 \\
\hline $122 \mathrm{a}$ & 122.52 & 122.6 \\
\hline $123 \mathrm{a}$ & 122.7 & 123 \\
\hline $123 \mathrm{~b}$ & 123 & 123.15 \\
\hline $123 \mathrm{c}$ & 123.25 & 123.44 \\
\hline $124 \mathrm{a}$ & 123.58 & 124 \\
\hline $124 \mathrm{~b}$ & 124.16 & 124.46 \\
\hline 125 & 124.6 & 125 \\
\hline $126 \mathrm{a}$ & 125 & 126 \\
\hline $127 \mathrm{a}$ & 126 & 126.5 \\
\hline $127 \mathrm{~b}$ & 127.1 & 127.55 \\
\hline $128 \mathrm{a}$ & 127.86 & 128 \\
\hline $128 \mathrm{~b}$ & 128.1 & 128.51 \\
\hline $129 \mathrm{a}$ & 128.8 & 128.95 \\
\hline $129 \mathrm{~b}$ & 129.2 & 129.55 \\
\hline $130 \mathrm{a}$ & 129.8 & 130 \\
\hline $130 \mathrm{~b}$ & 130.37 & 130.53 \\
\hline $130 \mathrm{c}$ & 130.67 & 130.83 \\
\hline $131 \mathrm{a}$ & 130.96 & 131 \\
\hline $131 \mathrm{~b}$ & 131.08 & 131.2 \\
\hline $131 \mathrm{c}$ & 131.48 & 131.57 \\
\hline $131 \mathrm{~d}$ & 131.69 & 131.78 \\
\hline $132 \mathrm{a}$ & 131.8 & 132 \\
\hline $132 \mathrm{~b}$ & 132 & 132.14 \\
\hline $132 \mathrm{c}$ & 132.39 & 132.56 \\
\hline $132 \mathrm{~d}$ & 132.6 & 132.73 \\
\hline $133 \mathrm{a}$ & 132.86 & 133 \\
\hline $133 \mathrm{~b}$ & 133 & 133.19 \\
\hline $133 \mathrm{c}$ & 133.35 & 133.58 \\
\hline $133 \mathrm{~d}$ & 133.58 & 133.72 \\
\hline $134 \mathrm{a}$ & 133.82 & 134 \\
\hline $134 \mathrm{~b}$ & 134 & 134.25 \\
\hline $134 \mathrm{c}$ & 134.51 & 134.81 \\
\hline $135 \mathrm{a}$ & 134.85 & 134.97 \\
\hline $135 \mathrm{~b}$ & 135 & 135.3 \\
\hline & 135.4 & 135.87 \\
\hline $136 \mathrm{~b}$ & 136.1 & 136.5 \\
\hline
\end{tabular}

Another indication of the accuracy of the array-element localization is obtained by noting that the array elements were spaced at $15-\mathrm{m}$ intervals along the cable; therefore, the interelement distances inferred from the acoustic localization should not exceed this value. In the approximately 30 array-shape determinations performed (60 if we count the $300-$ and $500-\mathrm{Hz}$ determinations separately), which collectively include about 900 (or 1800) interelement separations, only a few occurrences of separation estimates greater than $15 \mathrm{~m}$ for any nearest neighbor element pair were found; these values were typically $15.05 \mathrm{~m}$, a small violation of the distance constraint. By contrast, for each of the localizations, interelement separations between 14.5 and $15 \mathrm{~m}$ were the most commonly found values, an indication that the currents extended the array well, although not in a straight line. That interelement distances in the range of 14.5-15 m were usually obtained, while values exceeding $15 \mathrm{~m}$ (even by a small amount) were very rare is consistent with the smallness of the localization errors inferred from the $300-\mathrm{Hz} / 500-\mathrm{Hz}$ differences.

Another check of the array-element localization was available from results of two light bulb implosion runs, performed on JD 125 and JD 135. The JD 125 implosion data obtained from repeated light bulb deployments at two stations at about 2-km range and nearly orthogonal directions from the VLA site are compared in Fig. 21, the 300/224 Hz array-element localization results. Reasonable agreement is seen over the length of the array. Typical differences, too small to see on the scale of this plot, were on the order of $3 \mathrm{~m}$. The second light bulb localization run was made on JD 135 while the deployment ship ran a near-circular 2-km diameter track around the VLA, dropping light bulbs at nominal 100-m intervals. For this run, reasonable agreement between the methods is seen for about half of the array (Fig. 22). The origin of the discrepancy in the upper part (near the VLA) is being investigated.

In Table II, we list the 32 intervals for which array shape determinations have been made to date. We note that, while the array shape is often stable for several hours, it is seldom stationary for periods longer than $12 \mathrm{~h}$. The element positions, using the coordinate system defined in Fig. 20, are shown over a 16-d period in Fig. 9. Differences in positions obtained with the 300- and $500-\mathrm{Hz}$ data for the same interval are not visible on this scale. Since the positions are determined relative to array element 1 , this element is always found at $(0,0)$. The extreme motions are found near the center of the array, with the largest position differences determined to date measuring about $170 \mathrm{~m}$. We separate these data into four groups representing the experiment intervals JD 122-127, 127-130, 130-133, and 133-138 in Fig. 23(a)-(d). The motion shown in Fig. 23(a), representing the early part of the experiment, has a smaller amplitude (about $50 \mathrm{~m}$ maximum) than the motion observed in later phases. This smaller motion, suggesting smaller tidal and soliton currents, is consistent with lessdynamic temperature variations shown for JD122-127 in Fig. 9.

\section{ACKNOWLEDGMENT}

The following WHOI employees made significant contributions to the success of the experiment:

- J. Kemp and his mooring crew, who did a superb job outfitting three oceanographic ships and deploying the acoustic and physical oceanographic moorings;

- K. von der Heydt, who oversaw the array design, construction and deployment;

- T. Duda and J. Irish for the thermistor data taken at the source and receiver locations;

- A. Newhall, for his aid in accessing and understanding these data.

NRL personnel E. Carey, J. Schindall, and M. McCord all contributed to the acquisition and preparation of the NRL 300and $500-\mathrm{Hz}$ acoustic sources. The Asian Seas International Acoustics Experiment was an interinstitutional and multinational oceanography and ocean acoustics research effort. The South China Sea experiment was coordinated on the United States side by Dr. S. Ramp and Dr. C.-S. Chiu of the NPGS and Dr. J. Lynch of WHOI and on the Taiwan side by Dr. T. Yung Tang from the National Taiwan University. 


\section{REFERENCES}

[1] A. K. Liu, Y. S. Chang, M.-K. Hsu, and N. K. Liang, "Evolution of nonlinear internal waves in the East and South China Seas," J. Geophys. Res., vol. 103, pp. 7995-8008, 1998.

[2] Y. Y. Lin, J. F. Lynch, C. Nick, C. Chi-Fang, A. Newhall, A. Turgut, S. Schock, C. S. Chiu, L. Bartek, and C. S. Liu, "An estimate of the bottom compressional speed wave profile in the northeastern South China Sea using 'source of opportunity'," IEEE J. Oceanic Eng., vol. 29, pp. 1231-1248, Oct. 2004.

[3] A. Turgut, private communication Core analysis private communication, Naval Res. Lab., Washington, DC

[4] S. G. Schock, "Remote estimates of physical and acoustic sediment properties in South China sea using chirp sonar data and the Biot model," IEEE J. Oceanic Eng., vol. 29, pp. 1218-1230, Oct. 2004.

[5] G. Gawarkiewicz, J. Wang, M. Caruso, S. Ramp, K. Brink, and F. Bahr, "Shelfbreak circulation and thermohaline structure in the northern South China sea-Contrasting spring conditions in 2000 and 2001," IEEE J. Oceanic Eng., vol. 29, pp. 1131-1143, Oct. 2004.

[6] M. H. Orr and P. C. Mignerey, "Nonlinear internal waves in the South China Sea-Observation of the conversion of depression internal waves to elevation internal waves," J. Geophys. Res., vol. 108, no. C3, p. 3064, 2003

[7] T. Duda and J. Irish, private communication Experimental temperature data.

[8] J. S. Bendat and A. G. Piersol, Random Data Analysis and Measurement Procedures. New York: Wiley, 1986, p. 137.

[9] W. M. Carey, P. G. Cable, W. L. Siegmann, and J. F. Lynch, "Measurement of sound transmission and signal gain in the complex strait of Korea," IEEE J. Oceanic. Eng., vol. 27, pp. 841-852, Oct. 2002.

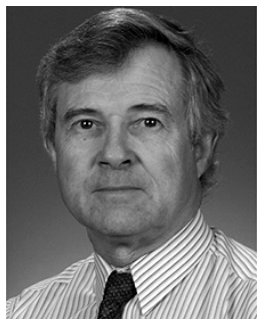

Marshall H. Orr received the Ph.D. degree in experimental nuclear structure physics from The Pennsylvania State University, State College, in December 1972.

He developed an interest in the fields of underwater acoustics, ocean acoustics, marine geophysics, and physical oceanography. He currently is a Branch Head in the Acoustics Division of the Naval Research Laboratory (NRL), Washington, DC. Recently, his research has addressed the physics of acoustic propagation in the random sound field of the littorals.

He has focused on relating acoustic signal variability to internal tide, internal wave, and fine-scale oceanography-induced sound-speed variability with a focus on the understanding the impact of the variability on array performance in the littorals. As part of his research interests, he uses a high-frequency acoustic flow-visualization instrumentation to visualize fine and small-scale fluid processes in the littoral waters.

Dr. Orr is a Fellow of the Acoustic Society of America and a Member of the American Geophysical Union.

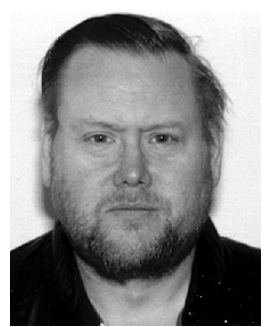

Bruce H Pasewark (A'99) received the B.S. and M.S. degrees in physics from the University of Virginia, Charlottesville, in 1975 and 1979, respectively, specializing in low-temperature thermodynamics, and the Ph.D. degree in applied marine physics from the University of Miami, Coral Gables, FL, in 2000.

From 1979 to 1982, he was a Research Associate in the Chemical Engineering Department, Texas A\&M University, College Station. Since 1982, he has been a Research Physicist in the Acoustics Division, Naval Research Laboratory, Washington, DC. In 1984, he was a Visiting Scientist with the Marine Physical Laboratory/Scripps Institute of Oceanography, University of California at San Diego, La Jolla, working on a shallow-water acoustic scattering project. He spent 1989 on assignment at the Rosenthiel School of Marine and Atmospheric Sciences, University of Miami, studying general underwater acoustics and oceanography. He has been Chief Scientist on several multinational, multilaboratory, multiship sea-going experiments and has participated in dozens of experiments in a slightly lesser roll. His primary research involves experimental underwater acoustics and signal processing in the shallow-water environment. His current research interests include acoustic propagation in a random medium and the dynamic relationship between acoustics and oceanography.
Stephen N. Wolf received the B.S. degree from Lebanon Valley College, Annville, PA, in 1966 and the Ph.D. degree from the University of Maryland, Baltimore County, in 1971

Since that time, he has been with the Naval Research Laboratory, Washington, DC, in the field of experimental shallow-water acoustics and acoustic modeling His research interests have included acoustic propagation, reverberation, ambient noise, target strength, random medium influences, and signal processing. From 1986 to 1988, he was a Visiting Scientist at the Defence Research Establishment Atlantic, Dartmouth, NS, Canada. He has authored 13 refereed journal articles and 22 conference proceedings and laboratory reports.

Dr. Wolf is a Fellow of the Acoustical Society of America.

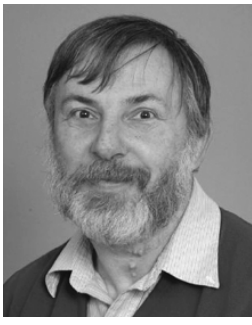

James F. Lynch (M'96-SM'03) was born in Jersey City, NJ, on June 3, 1950. He received the B.S. degree in physics from Stevens Institute of Technology, Hoboken, NJ, in 1972 and the Ph.D. degree in physics from the University of Texas, Austin, in 1978.

He was with the Applied Research Laboratories, University of Texas at Austin (ARL/UT) from 1978 to 1981 , after which he joined the scientific staff at the Woods Hole Oceanographic Institution (WHOI) Woods Hole, MA. He has been with WHOI since then and currently holds the position of Senior Scientist in the Applied Ocean Physics and Engineering Department. His research specialty areas are ocean acoustics and acoustical oceanography, but he also greatly enjoys occasional forays into physical oceanography, marine geology, and marine biology.

Dr. Lynch is a Fellow of the Acoustical Society of America and Editor-inChief of the IEEE JOURNAL OF OCEANIC ENGINEERING.

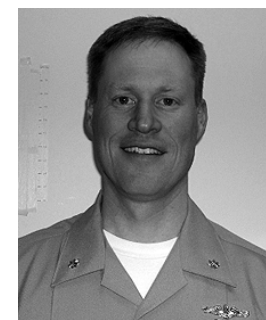

Theodore Schroeder was born in Wauseon, $\mathrm{OH}$ $\mathrm{He}$ was raised in St. Louis, MO, and graduated with B.S. degree (summa cum laude) in mechanical engineering from the University of Missouri-Rolla, in 1989. He earned the M.S. degree in oceanographic engineering from the Massachusetts Institute of Technology, Cambridge, MA, Woods Hole Oceanographic Institute/Woods Hole, MA, (MIT/WHOI), Joint Program in 2002.

After completing nuclear power training in 1991, he served as the Reactor Controls Assistant and Communicator at the USS Baltimore (SSN 704), Norfolk, VA. Following duty at the Office of Naval Intelligence, he graduated with distinction from the Submarine Officer Advanced Course, in 1997, and reported to USS Alaska (SSBN 732) (Gold) in Bangor, WA, as the Navigator. During this tour, he completed five Strategic Deterrent patrols and finished the tour as the OIC of the Gold Crew prior to crew combination in preparation for a D5 Trident Missile Backfit. In December 2002, he reported to the USS Parche (SNN 683) as the Executive Officer. In December 2004, he reported to the OPNAV staff for duty in the Submarine Modernization Branch.

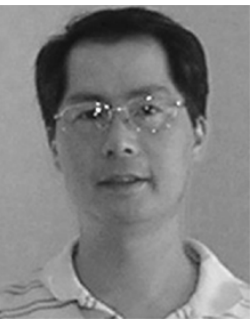

Ching-Sang Chiu received the $\mathrm{Ph} . \mathrm{D}$. degree from the Massachusetts Institute of Technology (MIT), Cambridge, and Woods Hole Oceanographic Institution (WHOI), Woods Hole, MA, joint program in 1985.

$\mathrm{He}$ is Professor of Oceanography at the Naval Postgraduate School, Washington, DC. His current research interests include shallow-water acoustics (particularly volume variability effects in shelfbreak regions, coastal ocean acoustic tomography, and passive detection) and localization of marine mammal vocalizations

Dr. Chiu is a Fellow of the Acoustical Society of America. 